
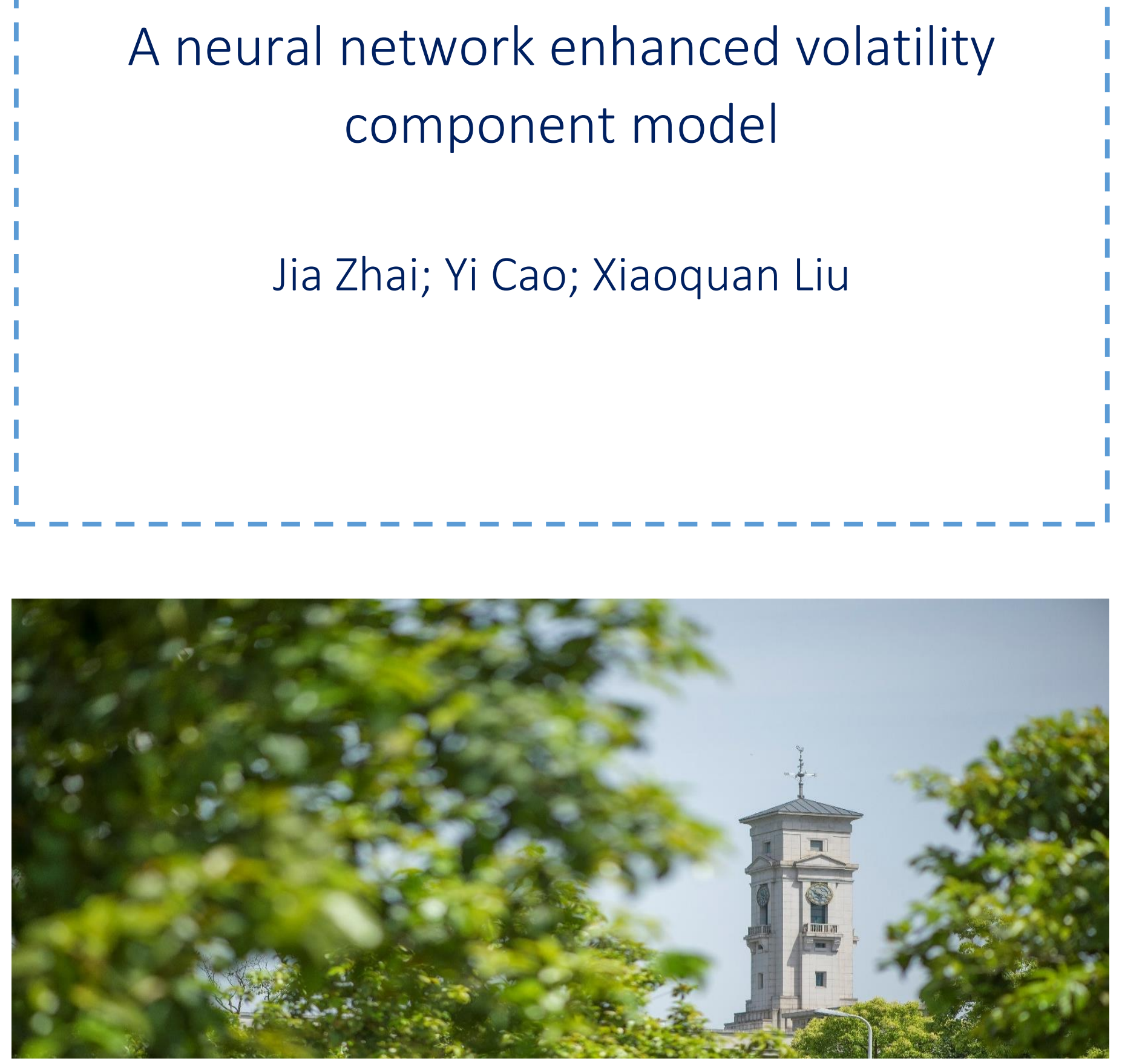
Faculty of Business, University of Nottingham Ningbo China, 199 Taikang East Road, Ningbo, 315100, Zhejiang, China.

First published 2020

This work is made available under the terms of the Creative Commons Attribution 4.0 International License:

http://creativecommons.org/licenses/by/4.0

The work is licenced to the University of Nottingham Ningbo China under the Global University Publication Licence:

https://www.nottingham.edu.cn/en/library/documents/researchsupport/global-university-publications-licence.pdf 


\title{
A neural network enhanced volatility component model ${ }^{*}$
}

\author{
Jia Zhai ${ }^{\dagger} \quad \mathrm{Yi} \mathrm{Cao}^{\ddagger} \quad$ Xiaoquan $\mathrm{Liu}^{\S}$
}

December, 2019

\begin{abstract}
Volatility prediction, a central issue in financial econometrics, attracts increasing attention in the data science literature as advances in computational methods enable us to develop models with great forecasting precision. In this paper, we draw upon both strands of the literature and develop a novel two-component volatility model. The realized volatility is decomposed by a nonparametric filter into long- and short-run components, which are modeled by an artificial neural network and an ARMA process, respectively. We use intraday data on four major exchange rates and a Chinese stock index to construct daily realized volatility and perform out-of-sample evaluation of volatility forecasts generated by our model and well-established alternatives. Empirical results show that our model outperforms alternative models across all statistical metrics and over different forecasting horizons. Furthermore, volatility forecasts from our model offer economic gain to a mean-variance utility investor with higher portfolio returns and Sharpe ratio.
\end{abstract}

JEL code: C63, F47

Keywords: Wavelet Analysis; ARMA Process; Volatility Prediction; Exchange Rates.

${ }^{*}$ We thank the Editor, Professor Jim Gatheral, and two anonymous referees for their helpful suggestions. We also thank Dr. Ying Jiang, participants at the China International Risk Forum in Shanghai Jiaotong University in 2017, and seminar participants at NYU Shanghai in 2018. All remaining errors are our own.

${ }^{\dagger}$ Salford Business School, University of Salford, Salford M5 4WT, UK. Email: j.zhai@salford.ac.uk.

${ }^{\ddagger}$ Management Science and Business Economics Group, University of Edinburgh Business School, 29 Buccleuch Place, Edinburgh EH8 9JS, UK. Email: jason.caoyi@gmail.com.

${ }^{\S}$ Corresponding author. Nottingham University Business School China, University of Nottingham Ningbo, Ningbo 315100, China. Email: xiaoquan.liu@nottingham.edu.cn. Phone: +8657488180000 ext 8207. 


\section{Introduction}

Volatility modeling and prediction play a crucial role in asset allocation, portfolio construction and risk management, as accurate volatility forecasts are of huge importance to traders, fund managers, and regulators. Traditional models are able to capture such stylized facts as volatility persistence and clustering, including the Autoregressive Conditional Heteroskedastic (ARCH) model of Engle (1982), the Generalized ARCH model of Bollerslev (1986), and the Autoregressive Fractionally Integrated Moving Average (ARFIMA) model of Granger (1980). Meanwhile, Taylor (1994) and Shephard (1996), among others, model volatility as an unobserved component following some latent stochastic process. These stochastic volatility models are theoretically well-founded and widely implemented.

More recently, volatility component models have attracted growing attention in the literature since the seminal work of Engle and Lee (1999). This formulation breaks the volatility dynamics into two additive components, a short-run and transitory component and a long-term and persistent one. This parsimonious parameterization is not only able to capture the complicated volatility dynamics but also capable of handling structural breaks in asset return volatility (Wang and Ghysels, 2015). Empirically, the two-component models generate more accurate forecasts than one-factor ones (Adrian and Rosenberg, 2008; Engle et al., 2013). Some component models are applied to rangebased volatility measures (Alizadeh et al., 2002; Harris et al., 2011) and asset return correlation (Colacito et al., 2011). Multiplicative component models have also been developed (Engle and Rangel, 2008; Engle and Sokalska, 2012).

Despite the empirical success of two-component volatility models, exactly what processes these two components follow is an open question. This leaves a lot of room for innovation. Some studies motivate the model via economic theories: Engle et al. (2013) link macroeconomic fundamentals to stock price volatility and incorporate macroeconomic variables in the long-term component specification, whereas Adrian and Rosenberg (2008) interpret the two components as asset pricing factors for financial constraint and business cycles, respectively, and model them both as a meanreverting $\mathrm{AR}(1)$ process. Others swap economic intuition for econometric flexibility such as Harris et al. (2011), which let the long-term component follow a random walk.

Our study is motivated by and contributes to this strand of the literature. Our first contribution is that methodologically we adopt powerful computational methods in developing a novel twocomponent volatility model. The long-run component is extracted from the realized volatility via 
the wavelet transform, a popular de-noising and approximation method in engineering, which has found its way to the economics and finance literature (see Esteban-Bravo and Vidal-Sanz, 2007; Haven et al., 2009, for example). The long-term component is then approximated by an artificial neural network due to its strong capability in capturing trend in the time series forecasting (Zhang and Qi, 2005). The neural networks are flexible, inherently nonlinear, and data-driven, and they have been increasingly utilized in the finance literature (see Breaban and Noussair, 2018; Garcia and Gencay, 2000; Hutchinson et al., 1994, for example), and in volatility forecasting in particular (Liu et al., 2018). The short-run component, the difference between realized volatilities and the long-term component, is obtained while ensuring its stationarity property.

We undertake a number of statistical metrics to evaluate the forecasting precision of our model against the component GARCH of Engle and Lee (1999) and the two-component model of Harris et al. (2011), which are directly comparable to our model. The data are 5-minute exchange rates for the EUR/USD, GBP/EUR, GBP/JPY, and GBP/USD from September 2009 to August 2015, and 5-minute CSI 300 index, a major stock index in the Chinese equity market, from August 2008 to September $2017 .^{1}$

Our second contribution is that empirically we show that our proposed model delivers significantly improved and robust out-of-sample forecasts. In the baseline forecasting exercises, our neural network enhanced volatility component model consistently dominates the competing models in producing more accurate volatility forecasts with smaller root mean square forecasting error (RMSFE), is always the preferred model in the Diebold and Mariano (1995) pairwise comparison, shows superior predictive ability in the Hansen (2005) test, and offers better interval forecasts in the likelihood ratio tests of Christoffersen (1998). This is the case over all forecasting horizons from one day up to 16 months ahead. ${ }^{2}$

What drives this superior performance? We analyze the forecasting errors separately for the short- and long-run components for the three models. Over short horizons of up to 20 days, the three models exhibit similar forecasting errors for the two components. Over longer horizons, the forecasting errors of the short-run components are still comparable across the three models. For the long-term component, the absolute percentage error (APE) is under $1.47 \%$ for our neural network enhanced model. However, the APE for the long-term component is between $16 \%$ and $25 \%$ for the

\footnotetext{
${ }^{1}$ Launched in April 2005, the CSI 300 index represents the most comprehensive and widely followed index in the Chinese stock market. The index is based on the largest and most liquid A-shares in the Shanghai and Shenzhen Stock Exchanges and re-balanced every six months.

${ }^{2}$ In the online appendix, we report statistical comparison between our model and the EGARCH model of Nelson (1991), the ARFIMA model of Granger (1980), and the HAR of Corsi (2009). The results are qualitatively the same.
} 
component GARCH, and between $17 \%$ and $33 \%$ for the Harris et al. (2011) model, representing a massive increase. In other words, our neural network enhance model is much better at capturing the long-term persistence and this is where the difference in the forecasting performance comes from. Hence, the artificial neural network method is the key to achieving prediction precision and underlines our contribution to the literature.

We perform a number of robustness tests along three directions. First, we re-run the forecasting evaluation between the models when volatility is computed as the intraday price range. The rangebased volatility measure has received renewed attention recently in the literature as it is shown to be more efficient than the squared return and more robust than realized volatility in handling market microstructure noise (Alizadeh et al., 2002; Brandt and Jones, 2006). Second, following Patton (2011) and Bollerslev et al. (2016a), we use QLIKE as the loss function when comparing volatility predictions. The ranking of the volatility models remains unchanged and our neural network enhanced model always comes out on top. Third, we use two popular recurrent deep neural network models, namely the long short-term memory (LSTM) model and the gated recurrent unit (GRU) model, to describe the long-term component and obtain qualitatively similar results.

As statistical significance does not necessarily translate into economic gains, we conduct a portfolio exercise to explore the economic value of the volatility forecasts. We assume that a meanvariance utility investor allocates her wealth between the risk-free asset and one of the foreign exchange rates or the stock index. We take historical average returns as returns to the assets and optimize over the weights at different risk aversion levels. Thus, the optimal weights as well as the overall portfolio performance are determined entirely by the volatility forecasts. We show that when we use forecasts from our model, the portfolios offer higher annualized returns, higher Sharpe ratio, and higher certainty equivalent return (CER) across a range of risk aversion levels and for all risky assets. This highlights the economic significance of the volatility forecasts generated by our model.

Finally, it is worth mentioning that our empirical results do not suggest that the proposed model would outperform any alternative models in terms of volatility prediction. Instead, our objective is to showcase the value of data science methods in addressing traditional research questions in finance. In our paper, we illustrate this by showing that a statistically motivated component volatility model, when coupled with the state-of-art neural networks for modeling the long-term component, generates improved volatility predictions in comparison to traditional models.

The rest of the paper is structured as follows. In Section 2, we outline our neural network 
enhanced component model for volatility in detail and introduce the evaluation metrics for the outof-sample forecasting exercises. Section 3 introduces the data. In Section 4 we discuss empirical results, provide robustness checks, and undertake a portfolio exercise to test the economic value of volatility forecasts. Finally, Section 5 concludes.

\section{Model specifications}

\subsection{The neural network enhanced volatility model}

In our neural network enhanced volatility component model, we assume that daily realized volatility follows a two-component process specified as follows:

$$
\begin{aligned}
\sigma_{t} & =L_{t}+S_{t}, \\
S_{t} & =c+\sum_{i=1}^{p} \varphi_{i} S_{t-i}+\sum_{j=1}^{q} \theta_{j} \varepsilon_{t-j}+\varepsilon_{t},
\end{aligned}
$$

where $\sigma_{t}$ is the realized volatility, $L_{t}$ and $S_{t}$ are the long- and short-run components of $\sigma_{t}$, respectively, at time $t, c$ is a constant, $\varphi_{i}$ and $\theta_{i}$ are model parameters, and $\varepsilon_{t}$ is the random error term with zero mean and constant variance. We assume that $L_{t}$ follows a smooth and non-stationary process but leave its precise dynamics unspecified. The short-term component $S_{t}$ is assumed to follow a stationary ARMA process.

We implement this two-component model in three steps. First, we extract the long-term component $L_{t}$ from the realized volatility via the wavelet method. The short-term component can subsequently be obtained as $S_{t}=\sigma_{t}-L_{t}$. In Step 2, to describe the dynamics of $L_{t}$ and forecast $n$-step ahead value $L_{t+n}$, we apply an autoregressive artificial neural network on $L_{t}$. The future value of $L_{t+n}$ can thus be predicted via the estimated neural network. In the final step, the shortterm component $S_{t}=\sigma_{t}-L_{t}$ is modeled by an $\operatorname{ARMA}(p, q)$ model. The $n$-step ahead value of $S_{t+n}$ is obtained via the estimated $\operatorname{ARMA}(p, q)$ model. This procedure allows us to forecast the $n$-step ahead future value of the volatility at time $t+n$ by $\sigma_{t+n}=L_{t+n}+S_{t+n}$. These three steps are elaborated below.

Step 1. Volatility decomposition

We adopt the wavelet analysis to extract the long-term component $L_{t}$, which has been successfully applied to different types of raw data, such as option prices (Haven et al., 2012) and exchange 
rates (Barunik et al., 2016). A key feature of the wavelet transform is that it can decompose any square integrable function into a combination of some scaling function and wavelet functions, each factored by their corresponding approximation coefficients and detail coefficients. Once the original function is decomposed, its detail coefficients can be utilized for de-noising via a hard or soft thresholding (Daubechies, 1992).

As discussed in Haven et al. (2012), the choice of the decomposition level is important for the de-noising effect. In Figure 1 we provide a comparison of the long-/short-term components by the wavelet transform at decomposition levels 7, 4, and 1 in the top, middle, and bottom panels, respectively. The data are daily realized volatility of EUR/USD from 27 Sept 2009 to 7 December 2012. We clearly observe that as the decomposition level decreases, the long-run component is less smooth and behaves more like the original volatility series whereas the short-term component looks more stationary, which is the assumption underlying volatility component models. To determine the appropriate decomposition level, we undertake the Augmented Dickey-Fuller (ADF) test (Fuller, 1976) with the null hypothesis that a unit root is present in the short-run component. ${ }^{3}$ We conduct the ADF test at every decomposition level from 7 to 1 . Once the null is rejected, the decomposition level is chosen. In Figure 1, the null is rejected at level 3 with a significant $p$-value of 0.01 .

\section{Step 2. Modeling the long-run component}

In the second step, an artificial autoregressive neural network (ARNN) is applied to the longterm component $L_{t}$. The ARNN has been applied to the time series modeling and shown to outperform traditional models such as the GARCH, EGARCH, and ARFIMA in volatility forecasting in the computer science literature (Kristjanpoller et al., 2014; Kristjanpoller and Minutolo, 2016), especially after the data are deseasonalized (Kristjanpoller and Minutolo, 2015). This is particularly true for the three-layer ARNN (Patil et al., 2008; Zhang and Qi, 2005), as the model exhibits an advantage over recurrent feed-forward neural network with less sensitivity to the problem of long-term dependence (Mustafaraj et al., 2011).

Motivated by this, we utilize an ARNN with three layers to model the long-term component $L_{t}$. The three layers are, respectively, an input layer that includes lagged $L_{t}$ inputs to the network; a hidden layer with hyperbolic tangential activation functions; and an output layers with a linear activation function. The model assumes the following general form for one-step ahead forecasts

\footnotetext{
${ }^{3}$ We choose the ADF test because it is widely used in the literature although it is not the most powerful.
} 
(Mustafaraj et al., 2011; Siegelmann et al., 1997):

$$
\begin{aligned}
\hat{L}_{t}\left(\theta_{A R N N}\right) & =g\left[\varphi_{i}(t), \theta_{A R N N}\right] \\
& =F_{j} \sum_{u=1}^{N_{h}} W_{j, u} f_{u}\left(\sum_{i=1}^{N_{u}} \varphi_{i}(t) w_{u, i}+w_{u, o}\right)+W_{j, o}
\end{aligned}
$$

where $g\left[\varphi_{i}(t), \theta_{A R N N}\right]$ is the ARNN function; $N_{h}$ is the number of hidden neurons; $N_{u}$ is the number of input variables; $W_{j, u}$ is the weight vector from the hidden neurons to the output layers; $w_{u, i}$ represents the matrix that contains the weight from the external input $N_{u}$ to the hidden neurons $N_{h} ; w_{u, o}$ and $W_{j, o}$ are the biases of hidden layers and the output layer, respectively, which can be interpreted as intercepts that must add up to one; $\varphi_{i}(t)$ is the vector that contains the input variables of the autoregressive part of the neural network; and $\theta_{A R N N}$ specifies the parameter vector, which contains all the adjustable parameters of the ARNN including the weights and the biases.

We follow the widely used configuration for the ARNN, where $f_{u}$ is the hyperbolic tangent function and $F_{j}$ is a linear function. Therefore, the forecasting is based on current value of the data at time $t$ as well as the stored data value at previous times, ie. $t-1, t-2, \ldots$ We select four input neurons and one hidden layer with 10 neurons to estimate the ARNN following the widely used configurations in Mandic and Chambers (2001), Mustafaraj et al. (2011), and Norgaard et al. (2000). ${ }^{4}$ This is a typical application of supervised-learning neural network, where the model parameters are obtained via the modified Levenberg-Marquardt algorithm (Hagan and Menhaj, 1994) to map the input variables to the output target variables.

To estimate the ARNN model, we extract the long-term component $L_{t}$ from the realized volatility in the training dataset by Step 1 and use the first $70 \%$ of data for training the ARNN model and the remained $30 \%$ for the validation process, in which the early-stop regularization is used to avoid overfitting. The training, including the validation, and testing of our proposed model are conducted via the rolling-forward procedure. The training, validation, and testing datasets are re-constructed each time as we move forward.

The one-step ahead forecast of $L_{t+1}$ is achieved by the current long-term component $L_{t}$ and the lagged values at $t-1, t-2$ and $t-3$. The multi-step ahead forecast is achieved by the closed-loop (Nrgaard et al., 2000). For example, to forecast $L_{t+2}$, we feed the predicted value $\hat{L}_{t+1}$ back to the

\footnotetext{
${ }^{4}$ As a robustness check, we have used 5 neurons and 15 neurons and obtain qualitatively similar results. These are available from the authors upon request.
} 
input of the ARNN model and shift the input vectors as $\left[\hat{L}_{t+1}, L_{t}, L_{t-1}, L_{t-2}\right]$. The value of $L_{t-3}$ is removed from the input vector. Once we obtain the predicted value of $\hat{L}_{t+2}$, we can forecast the $L_{t+3}$ by looping the $\hat{L}_{t+2}$ to the input vector.

\section{Step 3. Modeling the short-run component}

In the third step, we estimate an $\operatorname{ARMA}(p, q)$ model for the short-run component $\hat{S}_{t}=\sigma_{t}-L_{t}$. We implement the Bayesian Information Criterion (BIC) (Schwarz, 1978) to select the lag orders. Following the empirical studies in McQuarrie and Tsai (1998), we start with the ARMA(4,4) and estimate all $4 \times 4=16$ combinations of $p=1, \ldots, 4$ and $q=1, \ldots, 4$. To illustrate, for the data used in Figure 1, the BIC criteria suggests that $p=2$ and $q=2$. Hence, the $\operatorname{ARMA}(2,2)$ model is chosen and used for generating $n$-step ahead forecasts for the short-term component. Finally, the $n$-step ahead forecast of the realized volatility is the sum of the outputs from the ARNN and ARMA models according to Eq. (1).

\subsection{Forecast evaluation}

We estimate and compare the forecasting performance of three models: (1) our neural network enhanced model (Hybrid); (2) the component GARCH model of Engle and Lee (1999) (EL); and (3) the cyclical model of Harris et al. (2011) (HSY). We select these two models because they are popular members in the component volatility family and directly comparable to ours. In the online appendix, we also compare volatility forecasts generated by the EGARCH of Nelson (1991), the ARFIMA model of Granger (1980), and the HAR model of Corsi (2009), as they all show strong empirical performance in the literature.

Following Brandt and Jones (2006) and Harris et al. (2011), for point forecasts made at $t$, we construct average forecasts between $t+\tau_{1}$ and $t+\tau_{2}$ as follows:

$$
\hat{\sigma}_{t}\left(\tau_{1}, \tau_{2}\right)=\frac{1}{\tau_{2}-\tau_{1}+1} \sum_{\tau=\tau_{1}}^{\tau_{2}} \hat{\sigma}_{t+\tau}
$$

We consider a number of forecasting horizons with $\left(\tau_{1}, \tau_{2}\right)=(1,5),(1,20),(1,100),(100,200),(260,360)$, and $(400,500)$ days and report the average forecasts over these horizons. Hence, our forecast horizon ranges from 5 days to 16 months ahead. Notice that for $\left(\tau_{1}, \tau_{2}\right)=(100,200),(260,360)$, and $(400,500)$, we leave a gap of 100, 260, and 400 days before performing the forecasting exercise, representing a more challenging task for the models.

We adopt the following metrics to evaluate the forecasting performance of the volatility models. 
The root mean squared forecast error (RMSFE)

The RMSFE compares the forecasted volatility from a given model with the true volatility proxy and is computed as follows:

$$
\mathrm{RMSFE}=\sqrt{\frac{1}{R} \sum_{t=1}^{R}\left(\sigma\left(\tau_{1}, \tau_{2}\right)-\hat{\sigma}\left(\tau_{1}, \tau_{2}\right)\right)^{2}}
$$

where $R$ is the number of observations, and $\sigma\left(\tau_{1}, \tau_{2}\right)$ and $\hat{\sigma}\left(\tau_{1}, \tau_{2}\right)$ are the true volatility proxy and volatility forecasts, respectively.

The Diebold and Mariano (1995) statistic

We implement the pairwise comparison of Diebold and Mariano (1995) and test whether differences in RMSFE between two models are statistically significant. The test is defined as follows:

$$
\left[\left(\sigma_{t}\left(\tau_{1}, \tau_{2}\right)-\hat{\sigma}_{i, t}\left(\tau_{1}, \tau_{2}\right)\right)^{2}\right]-\left[\left(\sigma_{t}\left(\tau_{1}, \tau_{2}\right)-\hat{\sigma}_{j, t}\left(\tau_{1}, \tau_{2}\right)\right)^{2}\right]=d_{i, j}+\varepsilon_{t}\left(\tau_{1}, \tau_{2}\right)
$$

If $d_{i, j}$ is significantly greater than zero then model $j$ is preferred to model $i$, and vice versa.

$\underline{\text { The Superior Predictive Ability (SPA) test of Hansen (2005) }}$

To address the multiple-testing problem in the light of data mining, we conduct the SPA test of Hansen (2005). The null hypothesis states that the benchmark model is not inferior to any of the alternative models. A rejection of the null indicates that at least one competing model produces forecasts more accurate than the benchmark, which is the model with the lowest RMSFE. We report the stationary bootstrapped $p$-values obtained with 1000 replications for inference.

The interval forecast evaluation of Christoffersen (1998)

Christoffersen (1998) proposes metrics for evaluating the adequacy of the risk management measure Value-at-Risk (VaR). Since the VaR critically hinges upon volatility forecasts, the metrics can be readily implemented on volatility forecasts. The intuition is that the intervals around point volatility prediction should be narrow in tranquil times and wide in volatile times so that occurrences of volatility forecasts outside a pre-specified interval should be small and spread out over the sample and not come in clusters (Engle, 1982).

Based upon this intuition, let $B_{t \mid t-1}(m)$ and $U_{t \mid t-1}(m)$ denote the lower and upper limits of the ex ante interval forecast for time $t$ made at time $t-1$ for the coverage probability $m$. Christofferen 
(1998) defines the indicator variable $I_{t}$ as follows:

$$
I_{t}= \begin{cases}1, & \text { if } y_{t} \in\left[B_{t \mid t-1}(m), U_{t \mid t-1}(m)\right] \\ 0, & \text { if } y_{t} \notin\left[B_{t \mid t-1}(m), U_{t \mid t-1}(m)\right]\end{cases}
$$

where $\left\{y_{t}\right\}_{t=1}^{T}$ is a path of the time series $y_{t}$. The paper proves that a sequence of interval forecasts $\left\{B_{t \mid t-1}(m), U_{t \mid t-1}(m)\right\}_{t=1}^{T}$ exhibits correct conditional coverage if $\left\{I_{t}\right\}$ is independent and identically distributed and follows the Bernoulli distribution with parameter $m$ for all $t$, namely $\left\{I_{t}\right\} \stackrel{\text { iid }}{\sim} \operatorname{Bern}(m), \forall t$. This can be evaluated within a likelihood ratio testing framework that includes a test of unconditional coverage $L R_{u c}$, a test of independence $L R_{i n d}$, and a test of conditional coverage $L R_{c c}$.

For unconditional coverage, the null hypothesis that $E\left[I_{t}\right]=m$ is tested against the alternative $E\left[I_{t}\right] \neq m$. The likelihood ratio test between the null and the alternative can be expressed as follows:

$$
L R_{u c}=-2 \log \left[L\left(m ; I_{1}, I_{2}, \ldots, I_{T}\right) / L\left(\hat{\pi} ; I_{1}, I_{2}, \ldots, I_{T}\right)\right] \stackrel{\text { asy }}{\sim} \chi^{2}(1)
$$

where $L(m ; \cdot)$ and $L(\pi ; \cdot)$ are the likelihood under the null and the alternative hypotheses, respectively, and $\hat{\pi}=n_{1} /\left(n_{0}+n_{1}\right)$ is the maximum likelihood estimate of $\pi$.

The intuition for the independence test is that the zeros and ones should appear as a random process not in a time-dependent manner. Hence, independence is tested against the alternative of a first-order Markov process as follows:

$$
L R_{\text {ind }}=-2 \log \left[L\left(\hat{\Pi}_{2} ; I_{1}, I_{2}, \ldots, I_{T}\right) / L\left(\hat{\Pi}_{1} ; I_{1}, I_{2}, \ldots, I_{T}\right)\right] \stackrel{\text { asy }}{\sim} \chi^{2}(1)
$$

where

$$
\begin{gathered}
\hat{\Pi}_{1}=\left[\begin{array}{cc}
\frac{n_{00}}{n_{00}+n_{01}} & \frac{n_{01}}{n_{00}+n_{01}} \\
\frac{n_{10}}{n_{10}+n_{11}} & \frac{n_{11}}{n_{10}+n_{11}}
\end{array}\right], \\
\hat{\Pi}_{2}=\frac{n_{01}+n_{11}}{n_{00}+n_{10}+n_{01}+n_{11}},
\end{gathered}
$$

and $n_{i j}$ is the number of observations with value $i$ followed by $j$.

The tests for unconditional coverage and independence can be combined to form a complete test for conditional coverage that considers both correct coverage and the random sequence of 
occurrences as follows:

$$
L R_{c c}=-2 \log \left[L\left(m ; I_{1}, I_{2}, \ldots, I_{T}\right) / L\left(\hat{\Pi}_{1} ; I_{1}, I_{2}, \ldots, I_{T}\right)\right] \stackrel{\text { asy }}{\sim} \chi^{2}(2) .
$$

To summarize, the interval forecast evaluation captures the probability of unusually frequent consecutive exceedances thus offering additional insight into volatility forecasting violation and clustering. For example, if the probability of correct forecasting results over 100 days is $95 \%$, there should be less than five instances of consecutive exceedances. A low probability of the likelihood ratio tests implies repeated error and suggests model misspecification (Christoffersen and Pelletier, 2004).

\section{Data}

For exchange rates, our data are 5-minute data for EUR/USD, GBP/EUR, GBP/JPY, and GBP/USD from 27 September 2009 to 12 August 2015 with a total of 2.16 million observations over 2145 days. Data from 27 September 2009 to 7 December 2012 are used for the in-sample estimation and the rest for out-of-sample forecasts. For the CSI 300 index, the data are 5-minute index levels from 1 August 2005 to 29 September 2017 with 144,667 observations. The in-sample period is from 1 August 2005 to 31 July 2008 and the rest is for out-of-sample forecasting exercises. We aggregate intraday squared returns to obtain daily realized volatility as the proxy of the latent true volatility process following Andersen and Bollerslev (1998):

$$
\sigma_{t}^{2}=\sum_{n=1}^{N} r_{t, n}^{2}
$$

where $\sigma_{t}^{2}$ is the daily realized variance on day $t$, and $r_{t, n}^{2}$ is the squared logarithmic return on day $t$ for interval $n(n=1,2, \cdots, \mathrm{N})$.

Table 1 summarizes descriptive statistics for daily returns for the full sample. Panel A reports the mean, standard deviation, skewness and excess kurtosis while Panel B tabulates the autocorrelation coefficients and the Ljung and Box (1978) statistic for autocorrelation for the first five lags. The $p$-values for the Ljung-Box statistic are reported in parentheses. In the Ljung-Box test, the null hypothesis of no autocorrelation is strongly rejected. In Figure 2, we plot the autocorrelation of daily realized volatility for up to 30 lags for the assets. 


\section{Empirical analyses}

In Table 2, we summarize the RMSFE of realized volatility for the Hybrid, HSY, and EL models over six forecast horizons for all assets. We observe that in this comparison of point estimates, the Hybrid model shows very strong performance and dominates the other models in producing the smallest RMSFE, usually by a big margin, except for two occassions whereby the HSY is the bestperforming model for GBP/EUR over the shortest forecasting horizon and for the CSI index for the horizon between 1 and 100 days. Our results are consistent with evidence in the literature that the artificial neural network is able to produce significantly more accurate forecast when applied to deseasonalized data (Nelson et al., 1999; Zhang and Qi, 2005).

We conduct the Diebold and Mariano (1995) pairwise comparison between the forecasting difference of alternative volatility models and report the heteroscedasticity- and serial correlationadjusted $t$-statistics in Table 3 . A positive $t$-statistic indicates that the model in the row is preferred to the model in the column while a negative $t$-statistic suggests that the model in the column is preferred. We note that between the Hybrid and the HSY models, the Hybrid model is always preferred with significant $t$-statistic. It is often preferred when compared with the EL model, especially over longer horizons. The forecasting performances of the HSY and the EL models are similar on statistical terms.

We implement the superior predicative ability (SPA) test of Hansen (2005) and tabulate the stationary bootstrapped $p$-values, obtained via 1000 replications, in Table $4 .^{5}$ The null hypothesis that the benchmark model is not inferior to any of the competing models is resoundingly accepted with a high $p$-value when the Hybrid is the benchmark model. ${ }^{6}$

In Table 5, we undertake the likelihood ratio tests formulated in Christoffersen (1998) and report the $p$-values for the null hypotheses that at the $5 \%$ level the volatility forecasts exhibit the correct unconditional coverage $L R_{u c}$, are independent $L R_{i n d}$, and exhibit the correct conditional coverage $L R_{c c}$, respectively. Not surprisingly, the Hybrid model passes the test with flying colors and exhibits the highest $p$-values between 0.62 and 0.87 . This once again attests to the accuracy and adequacy of the volatility forecasts of our proposed model. For the HSY and EL models, the

\footnotetext{
${ }^{5}$ We aggregate volatility forecasts over all six horizons for each currency in order to provide a comprehensive and clear picture.

${ }^{6}$ We also conduct the Mincer and Zarnowitz (1969) regression, which shows the ability of the forecasted volatility in explaining the true volatility proxy. We find that the $R^{2}$ decreases massively with increasing forecast horizons for all models as expected. However, our proposed Hybrid model still shows $30 \%$ to $70 \%$ explanatory power for the longest forecast horizon and comes out stronger than the competing models. These results are available upon request from the authors.
} 
null is accepted with much lower $p$-values typically between 0.30 and 0.53 .

To summarize, in our baseline analysis we conduct a number of statistical tests to evaluate the out-of-sample performance of our neural network enhanced model and alternative two-component models. Our proposed model substantially outperforms the others in these econometric tests.

\subsection{Forecasting error analysis}

What drives the superior forecasting performance of our proposed model? To obtain a better understanding of the differences in the empirical performance between the models, we break down and analyze the forecasting errors for each component across the three models.

In Figure 3, we plot in Panels (a) and (b) the average absolute percentage error (APE) of the short- and long-term components, respectively, for EUR/USD for the three models. In Panel (a), the Hybrid model exhibits the smallest forecasting error for the short-term component, followed by the EL model, and the HSY on average offers the largest forecasting errors. Nevertheless, the errors for the short-term component are not too different from each other across the models and typically lie between $10 \%$ and $35 \%$. However, Panel (b) shows a very different pattern. Over the two shortest horizons of up to 20 days, all models produce quite accurate forecasts and the APE is less than 1\%. But as we move to longer horizons, we see massive difference between the APE for the long-term component: for the Hybrid model the APE is less than 2\%, whereas for the EL and the HSY, it goes up to $23 \%$ and $28 \%$, respectively. So it is the precision of the long-term component that drives the distinct performance of the models and this underlines the prowess of the neural network in capturing the trend component in time series of data. ${ }^{7}$

In Table 6, we report the average APE in percent for all test assets. The same pattern emerges that our neural network enhanced model shows extraordinary ability in capturing the trend and producing average APE of $1.47 \%$ at most even for the 16-month horizon. For the other two models, the average APE is somewhere between $15 \%$ and $32 \%$ for the long-term component over the longest forecasting horizon.

\subsection{Robustness check}

\section{Alternative volatility proxy}

We perform a number of robustness tests to show that our baseline results are not due to

\footnotetext{
${ }^{7}$ It is worth noting that the artificial neural network does not work well on modeling and forecasting the overall volatility dynamics. See Yao et al. (2017) for example.
} 
specific modeling choices. First, instead of using realized volatility we adopt the intraday rangebased measure for volatility modeling and as the proxy for true volatility. The range-based measure is proven robust to microstructure noise and receives renewed interest in the literature in recent years (Alizadeh et al., 2002; Engle and Gallo, 2006). It is specified as follows:

$$
\sigma_{t}^{2}=\frac{1}{4 \ln 2}\left(P_{t}^{H}-P_{t}^{L}\right)^{2}
$$

where $P_{t}^{H}$ and $P_{t}^{L}$ are the highest and lowest prices on day $t$, respectively.

Table A1 in the online appendix reports the summary statistics for the daily range-based volatility for the full sample. In Table 7, we tabulate the $p$-values for the SPA test when volatilities are measured as the squared range between the highest and lowest prices in a day. We observe the same pattern that the Hybrid model is not inferior to the competing HSY and EL models with similarly high $p$-values as those obtained in our baseline results in Table 4.

\section{Alternative loss function for volatility prediction accuracy}

Our second robustness check is to adopt the loss function, QLIKE, between the true and forecasted volatility. It is defined as follows:

$$
Q L I K E=\log \hat{\sigma}^{2}+\frac{\sigma^{2}}{\hat{\sigma}^{2}}
$$

where $\hat{\sigma}^{2}$ and $\sigma^{2}$ are variance forecasts and the true variance proxy, respectively. This particular loss function, robust when the proxy is unbiased but imperfect, is recommended in Patton (2011) and Bollerslev et al. (2016b). In Table 8, we report the heteroscedasticity- and serial correlationadjusted $t$-statistic for the Diebold and Mariano (1995) pairwise comparison with $Q L I K E$ as the loss function. We find that at the Hybrid model is always preferred to the HSY and EL models. We further conduct the SPA test of Hansen (2005) on range-based volatility with the QLIKE as the loss function and summarize the results in Table 9 . We obtain qualitatively similar results to the baseline findings. The null hypothesis is always accepted with very high $p$-value.

\section{Alternative model for the long-term component}

Our paper proposes a framework in which adopting advanced neural network models for describing the long-term component helps generate more precise volatility forecasts. Hence, the volatility forecasting accuracy is not expected to be greatly affected by the specific neural network model we choose. In the third robustness test, we implement two popular neural network models, i.e., 
the long short-term memory model (LSTM) (Gers et al., 2000; Hochreiter and Schmidhuber, 1997) and the gated recurrent unit (GRU) model (Cho et al., 2014; Chung et al., 2014) for the long-term component, and call them the Hybrid-LSTM and Hybrid-GRU, respectively. The forecasting errors for these models are summarized in the last two columns in Table 2, and the Diebold and Mariano (1995) pairwise comparison results are reported in Table 10. Consistent with our conjecture, performance of these three hybrid models are statistically comparable as the $t$-statistic is always insignificant.

To summarize, the empirical results in the robustness tests further corroborate the baseline findings that the neural network enhanced volatility model outperforms the two-component models of Harris et al. (2011) and Engle and Lee (1999) in producing more accurate volatility predictions over different horizons for all test assets. ${ }^{8}$ This attests to the validity of proposed framework that neural network enhanced component volatility models generate more precise volatility predictions.

\subsection{Economic value of volatility forecasts}

A strong statistical performance does not necessarily indicate superior economic significance in the out-of-sample exercise. Therefore we analyze the economic value of volatility forecasts assuming a mean-variance utility investor who allocates her wealth between a risk-free asset and one of the four exchange rates or the stock index. We follow Wang et al. (2016) to construct the utility function as follows:

$$
U_{t}\left(r_{t}\right)=E_{t}\left(w_{t} r_{t}+r_{t, f}\right)-\frac{1}{2} \gamma \sigma_{t}^{2}\left(w_{t} r_{t}+r_{t, f}\right)
$$

where $w_{t}$ is the weight of the risky asset in the portfolio, $r_{t}$ is the return to the risky asset in excess of the risk-free rate, $r_{t, f}$, and $\gamma$ denotes the level of risk aversion. We maximize the utility function $U_{t}\left(r_{t}\right)$ with respect to the weight $w_{t}$ and obtain the ex ante optimal weight on day $t+1$ :

$$
\hat{w}_{t}=\frac{1}{\gamma}\left(\frac{\hat{r}_{t+1}}{\hat{\sigma}_{t+1}^{2}}\right),
$$

where $\hat{r}_{t+1}$ and $\hat{\sigma}_{t+1}^{2}$ are the mean and volatility forecasts, respectively, of the excess returns. In our study, risk-free rates come from the three-month US Treasury bill or the three-month Chinese national bond yield.

\footnotetext{
${ }^{8}$ We have conducted yet another robustness check by adopting the low-pass Hodrick and Prescott (1997) filter and see whether the performance of the Hybrid model is due to our choice of the particular decomposition method, i.e. the wavelet method. We obtain qualitatively similar results that the model with the Hodrick and Prescott (1997) filtering, with the long-/short-term component still modelled via the artificial neural network and the ARMA process, respectively, outperforms the HSY and EL models. The results are available from the authors upon request.
} 
Following Rapach et al. (2010) and Wang et al. (2016), we use the historical average as the mean forecasts for returns, $\hat{r}_{t+1}=\sum_{j=1}^{t} r_{j}$. Hence, for each level of risk aversion $\gamma$, the optimal weight $\hat{w}_{t}=\frac{1}{\gamma}\left(\frac{\hat{r}_{t+1}}{\hat{\sigma}_{t+1}^{2}}\right)$ of the portfolio is only determined by the volatility forecasts as different strategies share the same mean forecasts of returns. We use the Sharpe ratio (SR):

$$
\mathrm{SR}=\frac{\bar{\mu}_{p}}{\bar{\sigma}_{p}}
$$

and the certainty equivalent return $(\mathrm{CER})$ :

$$
\mathrm{CER}=\hat{\mu}_{p}-\frac{\gamma}{2} \hat{\sigma}_{p}^{2}
$$

to evaluate the performance of a portfolio, where $\bar{\mu}_{p}$ and $\bar{\sigma}_{p}$ are the mean and standard deviation of portfolio excess returns, respectively; $\hat{\mu}_{p}$ and $\hat{\sigma}_{p}^{2}$ are the mean and variance of portfolio returns in the out-of-sample period, respectively. For robustness, we adopt $\gamma=3,6$, and 9 to represent different level of risk aversion.

In Table 11, we report the annualized average return, the Sharpe ratio, and the CER of the portfolios constructed using realized volatility from different models. When the investor is assumed to have a relatively low level of risk aversion at $\gamma=3$, portfolios are able to achieve an annual return of $6.63 \%$ when volatility forecasts are generated by the Hybrid model for EUR/USD, and the Sharpe ratio is 0.37 , while the certainty equivalent return is slightly higher than $3 \%$. These are evidently higher than when the forecasts are generated by the competing models. As the investor becomes more risk averse with larger $\gamma$ value, she assigns a greater weight to the risk-free asset. This results in lower portfolio return, Sharpe ratio and the CER. Nevertheless, the same pattern exists that the portfolios formed using the Hybrid volatility forecasts offer higher return and risk-adjusted return, substantiating the economic value of the volatility forecasts from our proposed model.

Meanwhile, given the inextricable link between the macroeconomy and financial market volatility, especially the long-term component of the volatility dynamics (see Bloom, 2009; Chiu et al., 2018; Conrad et al., 2014; Engle et al., 2013, for example), we perform a vector autoregression to explore how the macroeconomic conditions impact on the persistent component of exchange rates. In particular, we examine the impulse response of the long-term component of EURUSD and GBPUSD to changes in two important US macroeconomic variables: the GDP growth and the Federal fund rate. We find that for both exchange rates, the long-term volatility trends down given a disturbance to the GDP growth over the next five months but picks up in the medium run, 
whereas a shock to the Federate fund rate increases the long-term component of volatility over the next five to ten months. ${ }^{9}$

\section{Conclusion}

The evidence that conditional volatility dynamics comprises both a long-term trend component and a strongly oscillating short-run component has crucial implication for volatility forecasting over both short and long horizons. In this paper, we build upon and extend the two-component volatility literature and develop a novel neural network enhanced volatility component model. We first decompose the daily realized volatility into long- and short-run components using the wavelet transform while implementing the ADF test to choose appropriate parameters and ensuring the stationarity assumption for the short-run component is satisfied. We then separately model the long- and short-run components with the artificial neural network and an ARMA model, respectively. The model is empirically evaluated using data on four exchange rates and a stock index over a number of forecast horizons. We compare the RMSFE and perform statistical evaluations such as the Diebold and Mariano (1995) test, the SPA test of Hansen (2005), and the interval forecast evaluation of Christoffersen (1998). In the out-of-sample comparison of volatility predictions generated by our proposed model and popular alternative models, we provide strong and robust evidence that our neural network enhanced model significantly outperforms the competing models. In economic terms, the volatility forecasts from the Hybrid model offers improved economic value to a mean-variance utility investor.

\section{Appendix}

\section{Specifications for competing volatility models}

Two-component model of Harris et al. (2011)

In Harris et al. (2011), the volatility follows a two-factor process specified as follows:

$$
\sigma_{t}=q_{t}+\alpha\left(\sigma_{t-1}-q_{t-1}\right)+\epsilon_{t}
$$

\footnotetext{
${ }^{9}$ These results are not reported to conserve space. They are available upon request from the authors.
} 
where $q_{t}$ is the long-run trend component of the volatility, the short-term component is defined as $\sigma_{t}-q_{t}$, and $\epsilon_{t}$ is a random error term with zero mean and constant variance. The model assumes the long-run trend follows a random walk over the forecast horizon so that $E\left[q_{t+i} \mid t\right]=q_{t}$ for all $i>0$. The two components are obtained via two nonparametric filters: the low-pass filter of Hodrick and Prescott (1997) and the band-pass filter of Christiano and Fitzgerald (2003).

\section{The Component GARCH model}

The two-component volatility model of Engle and Lee (1999) for returns over time period $t, r_{t}$, is given as follows:

$$
\begin{aligned}
r_{t} & =\sqrt{h_{t}} \epsilon_{t} \\
h_{t} & =\tau_{t}+g_{t} \\
\tau_{t} & =\omega+\rho \tau_{t-1}+\phi\left(r_{t-1}^{2}-h_{t-1}\right) \\
g_{t} & =\alpha\left(r_{t-1}^{2}-\tau_{t-1}\right)+\beta g_{t-1},
\end{aligned}
$$

where $\epsilon_{t}$ is an i.i.d series of standardized random variables. The variance $h_{t}$ is decomposed into $\tau_{t}$, the trend component, and $g_{t}$, the transitory component; and $\omega, \rho, \alpha$, and $\phi$ are model coefficients. 


\section{References}

Adrian, T., Rosenberg, J., 2008. Stock returns and volatility: Pricing the short-run and long-run components of market risk. Journal of Finance 63, 2997-3030.

Alizadeh, S., Brandt, M., Diebold, F., 2002. Range-based estimation of stochastic volatility models. Journal of Finance 57, 1047-1091.

Andersen, T. G., Bollerslev, T., 1998. Answering the skeptics: Yes, standard volatility models do provide accurate forecasts. International Economic Review 39, 885-905.

Barunik, J., Krehlik, T., Vacha, L., 2016. Modeling and forecasting exchange rate volatility in time-frequency domain. European Journal of Operational Research 251, 329-340.

Bloom, N., 2009. The impact of uncertainty shocks. Econometrica 77, 623-685.

Bollerslev, T., 1986. Generalized autoregressive conditional heteroskedasticity. Journal of Econometrics 31, 307-327.

Bollerslev, T., Li, S. Z., Todorov, V., 2016a. Roughing up beta: Continuous versus discontinuous betas and the cross section of expected stock returns. Journal of Financial Economics 120, 464490.

Bollerslev, T., Patton, A. J., Quaedvlieg, R., 2016b. Exploiting the errors: A simple approach for improved volatility forecasting. Journal of Econometrics 192, 1-18.

Brandt, M. W., Jones, C. S., 2006. Volatility forecasting with range-based EGARCH models. Journal of Business \& Economic Statistics 24, 470-486.

Breaban, A., Noussair, C. N., 2018. Emotional state and market behavior. Review of Finance 22, 279-309.

Chiu, C.-W. J., Harris, R. D., Stoja, E., Chin, M., 2018. Financial market volatility, macroeconomic fundamentals and investor sentiment. Journal of Banking \& Finance 92, 130-145.

Cho, K., Van Merriënboer, B., Bahdanau, D., Bengio, Y., 2014. On the properties of neural machine translation: Encoder-decoder approaches, arXiv preprint arXiv: 1409.1259.

Christiano, L., Fitzgerald, T., 2003. The band pass filter. International Economic Review 44, 435465. 
Christoffersen, P. F., 1998. Evaluating interval forecasts. International Economic Review 39, 841862.

Christoffersen, P. F., Pelletier, D., 2004. Backtesting Value-at-Risk: A duration-based approach. Journal of Financial Econometrics 2, 84-108.

Chung, J., Gulcehre, C., Cho, K., Bengio, Y., 2014. Empirical evaluation of gated recurrent neural networks on sequence modeling, arXiv preprint arXiv: 1412.3555.

Colacito, R., Engle, R. F., Ghysels, E., 2011. A component model for dynamic correlations. Journal of Econometrics 164, 45-59.

Conrad, C., Loch, K., Rittler, D., 2014. On the macroeconomic determinants of long-term volatilities and correlations in US stock and crude oil markets. Journal of Empirical Finance 29, 26-40.

Corsi, F., 2009. A simple approximate long-memory model of realized volatility. Journal of Financial Econometrics 7, 174-196.

Daubechies, I., 1992. Ten Lectures on Wavelets. Philadelphia: Socienty for Industrial and Applied Mathematics.

Diebold, F., Mariano, R. S., 1995. Comparing predictive accuracy. Journal of Business \& Economic Statistics 13, 253-263.

Engle, R., 1982. Autoregressive conditional heteroscedasticity with estimates of the variance of United Kingdom inflation. Econometrica 50, 987-1007.

Engle, R. F., Gallo, G. M., 2006. A multiple indicators model for volatility using intra-daily data. Journal of Econometrics 131, 3-27.

Engle, R. F., Ghysels, E., Sohn, B., 2013. Stock market volatility and macroeconomic fundamentals. Review of Economics and Statistics 95, 776-797.

Engle, R. F., Lee, G., 1999. A long-run and short-run component model of stock return volatility. In: Engle, R., White, H. (Eds.), Cointegration, Causality, and Forecasting: A Festschrift in Honour of Clive WJ Granger. Oxford University Press, pp. 475-497.

Engle, R. F., Rangel, J. G., 2008. The spline-GARCH model for low-frequency volatility and its global macroeconomic causes. Review of Financial Studies 21, 1187-1222. 
Engle, R. F., Sokalska, M. E., 2012. Forecasting intraday volatility in the US equity market. Multiplicative component GARCH. Journal of Financial Econometrics 10, 54-83.

Esteban-Bravo, M., Vidal-Sanz, J. M., 2007. Computing continuous-time growth models with boundary conditions via wavelets. Journal of Economic Dynamics \& Control 31, 3614-3643.

Fuller, W. A., 1976. Introduction to Statistical Time Series. John Wiley \& Sons.

Garcia, R., Gencay, R., 2000. Pricing and hedging derivative securities with neural networks and a homogeneity hint. Journal of Econometrics 94, 93-115.

Gers, F., Schmidhuber, J., Cummins, F., 2000. Learning to forget: Continual prediction with LSTM. Neural computation 12, 24-51.

Granger, C. W. J., 1980. Long memory relationships and the aggregation of dynamic models. Journal of Econometrics 14, 227-238.

Hagan, M., Menhaj, M., 1994. Training feedforward networks with the Marquardt algorithm. IEEE Transactions on Neural Networks 5, 989-993.

Hansen, P. R., 2005. A test of superior predictive ability. Journal of Business \& Economic Statistics $23,365-380$.

Harris, R., Stoja, E., Yilmaz, F., 2011. A cyclical model of exchange rate volatility. Journal of Banking \& Finance 35, 3055-3064.

Haven, E., Liu, X., Ma, C., Shen, L., 2009. Revealing the implied risk-neutral MGF from options: The wavelet method. Journal of Economic Dynamics \& Control 33, 692-709.

Haven, E., Liu, X., Shen, L., 2012. De-noising option prices with the wavelet method. European Journal of Operational Research 222, 104-112.

Hochreiter, S., Schmidhuber, J., 1997. Long short-term memory. Neural computation 9, 1735-1780.

Hodrick, R., Prescott, E., 1997. Post-war US business cycles: An empirical investigation. Journal of Money, Credit and Banking 29, 1-16.

Hutchinson, J. M., Lo, A., Poggio, T., 1994. A nonparametric approach to pricing and hedging derivative securities via learning networks. Journal of Finance 49, 851-889. 
Kristjanpoller, W., Fadic, A., Minutolo, M. C., 2014. Volatility forecast using hybrid neural network models. Expert Systems with Applications 41, 2437-2442.

Kristjanpoller, W., Minutolo, M., 2016. Forecasting volatility of oil price using an artificial neural network-GARCH model. Expert Systems with Applications 65, 233-241.

Kristjanpoller, W., Minutolo, M. C., 2015. Gold price volatility: A forecasting approach using the artificial neural network-GARCH model. Expert Systems with Applications 42, 7245-7251.

Liu, F., Pantelous, A. A., Von Mettenheim, H.-J., 2018. Forecasting and trading high frequency volatility on large indices. Quantitative Finance 18, 737-748.

Ljung, G. M., Box, G. E. P., 1978. On a measure of a lack of fit in time series models. Biometrika 65, 297-303.

Mandic, D., Chambers, J., 2001. Recurrent Neural Networks for Prediction. John Wiley \& Sons.

McQuarrie, A. D., Tsai, C.-L., 1998. Regression and Time Series Model Selection. Singapore: World Scientific.

Mincer, J., Zarnowitz, V., 1969. The evaluation of economic forecasts. In: Zarnowitz, J. (Ed.), Economic Forecasts and Expectations. National Bureau of Economic Research, New York.

Mustafaraj, G., Lowery, G., Chen, J., 2011. Prediction of room temperature and relative humidity by autoregressive linear and nonlinear neural network models for an open office. Energy and Buildings 43, 1452-1460.

Nelson, D., 1991. Conditional heteroskedasticity in asset returns: A new approach. Econometrica $59,347-370$.

Nelson, M., Hill, T., Remus, W., O’Conner, M., 1999. Time series forecasting using NNs: Should the data be deseasonalized first? Journal of Forecasting 18, 359-367.

Norgaard, M., Rawn, O., Poulsen, N., Hansen, L., 2000. Neural Network for Modeling and Control of Dynamic Systems. Springer-Verlag.

Nrgaard, M., Ravn, O. E., Poulsen, N. K., Hansen, L. K., 2000. Neural Networks for Modelling and Control of Dynamic Systems: A Practitioner's Handbook, 1st Edition. Springer-Verlag, Berlin, Heidelberg. 
Patil, S., Tantau, H., Salokhe, V., 2008. Modelling of tropical greenhouse temperature by autoregressive and neural network models. Biosystems Engineering 99, 423-431.

Patton, A., 2011. Volatility forecast comparison using imperfect volatility proxies. Journal of Econometrics 160, 246-256.

Rapach, D. E., Strauss, J. K., Zhou, G., 2010. Out-of-sample equity premium prediction: Combination forecasts and links to the real economy. Review of Financial Studies 23, 821-862.

Schwarz, G., 1978. Estimating the dimension of a model. Annals of Statistics 6, 461-464.

Shephard, N., 1996. Statistical aspects of ARCH and stochastic volatility. In: Cox, D. R., Hinkley, D. V., Barndorff-Nielsen, O. E. (Eds.), Time Series Models: In econometrics, finance and other applications. Chapman \& Hall/CRC Monographs on Statistics and Applied Probability, London, pp. $1-68$.

Siegelmann, H., Horne, B., Giles, C., 1997. Computational capabilities of recurrent NARX neural networks. IEEE Transactions on Systems, Man, and Cybernetics, Part B(Cybernetics) 27, 208215.

Taylor, S. J., 1994. Modelling stochastic volatilitiy: A review and comparative study. Mathematical Finance 4, 183-204.

Wang, F., Ghysels, E., 2015. Econometric analysis of volatility component models. Econometric Theory 31, 362-393.

Wang, Y., Ma, F., Wei, Y., Wu, C., 2016. Forecasting realized volatility in a changing world: A dynamic model averaging approach. Journal of Banking \& Finance 64, 136-149.

Yao, Y., Zhai, J., Cao, Y., Ding, X., Liu, J., Luo, Y., 2017. Data analytics enhanced component volatility model. Expert Systems with Applications 84, 232-241.

Zhang, P., Qi, M., 2005. Neural network forecasting for seasonal and trend time series. European Journal of Operational Research 160, 501-514. 
Table 1. Descriptive statistics for daily returns

This table summarizes the mean, standard deviation, skewness, excess kurtosis, and autocorrelations for daily returns to exchange rates and the stock index. The $p$-values for the Ljung-Box test are reported in parentheses. The sample period is from 27 Sept 2009 to 12 Aug 2015 for the exchange rates and from 1 August 2005 to 29 September 2017 for the CSI 300 index.

Panel A. Summary statistics

\begin{tabular}{lrrrr}
\hline Currency & Mean & Stdev & Skewness & Excess Kurt \\
\hline EUR/USD & $-1.4660 \mathrm{E}-04$ & $5.4663 \mathrm{E}-03$ & -0.0373 & 1.4210 \\
GBP/EUR & $1.4390 \mathrm{E}-04$ & $4.5858 \mathrm{E}-03$ & 0.1506 & 1.8071 \\
GBP/JPY & $1.7240 \mathrm{E}-04$ & $6.5854 \mathrm{E}-03$ & 0.5001 & 4.5186 \\
GBP/USD & $-7.7000 \mathrm{E}-05$ & $4.6281 \mathrm{E}-03$ & -0.0940 & 1.3284 \\
CSI 300 & $1.4400 \mathrm{E}-02$ & $9.5900 \mathrm{E}-03$ & 2.6100 & 14.500 \\
\hline
\end{tabular}

Panel B. Autocorrelation

\begin{tabular}{lrrrrrr}
\hline Currency & Lag 1 & Lag 2 & Lag 3 & Lag 4 & Lag 5 & Ljung-Box test \\
\hline EUR/USD & $3.62 \mathrm{E}-02$ & $-1.13 \mathrm{E}-02$ & $-2.67 \mathrm{E}-02$ & $-9.63 \mathrm{E}-03$ & $1.24 \mathrm{E}-03$ & $2.4200(0.1200)$ \\
GBP/EUR & $2.65 \mathrm{E}-02$ & $-4.68 \mathrm{E}-02$ & $-6.74 \mathrm{E}-02$ & $7.64 \mathrm{E}-04$ & $-1.62 \mathrm{E}-02$ & $1.2900(0.2560)$ \\
GBP/JPY & $2.80 \mathrm{E}-04$ & $-3.00 \mathrm{E}-02$ & $-6.40 \mathrm{E}-02$ & $1.28 \mathrm{E}-04$ & $5.03 \mathrm{E}-02$ & $0.0014(0.9900)$ \\
GBP/USD & $-2.57 \mathrm{E}-02$ & $-3.77 \mathrm{E}-02$ & $7.55 \mathrm{E}-03$ & $2.20 \mathrm{E}-03$ & $-1.38 \mathrm{E}-02$ & $1.2100(0.2710)$ \\
CSI 300 & $7.24 \mathrm{E}-01$ & $6.72 \mathrm{E}-01$ & $6.37 \mathrm{E}-01$ & $6.18 \mathrm{E}-01$ & $5.87 \mathrm{E}-01$ & $7160(0.2200)$ \\
\hline
\end{tabular}


Table 2. The forecasting accuracy of alternative volatility models

This table reports the root mean squared forecasting error (RMSFE) for the neural network enhanced model (Hybrid), the cyclical model of Harris et al. (2011) (HSY), and the component GARCH of Engle and Lee (1999)(EL). The RMSFE is also reported when the long-term component is modeled by the LSTM model (Hybrid-LSTM) or the GRU model (Hybrid-GRU). The out-of-sample period is from 8 December 2012 to 15 August 2015 for exchange rates, and from 1 August 2008 to 29 September 2017 for the CSI 300 index. The forecasting horizon is from day $t+\tau_{1}$ to day $t+\tau_{2}$.

\begin{tabular}{|c|c|c|c|c|c|c|c|}
\hline$\tau_{1}$ & $\tau_{2}$ & Currency & Hybrid & HSY & $\mathrm{EL}$ & Hybrid-LSTM & Hybrid-GRU \\
\hline \multirow[t]{5}{*}{1} & \multirow[t]{5}{*}{5} & EUR/USD & $1.9472 \mathrm{E}-04$ & $3.7272 \mathrm{E}-04$ & $5.3433 \mathrm{E}-04$ & $1.9023 \mathrm{E}-04$ & $1.8931 \mathrm{E}-04$ \\
\hline & & GBP/EUR & $1.7448 \mathrm{E}-04$ & $1.4507 \mathrm{E}-04$ & $9.3405 \mathrm{E}-04$ & $1.6747 \mathrm{E}-04$ & $1.6762 \mathrm{E}-04$ \\
\hline & & GBP/JPY & $1.8910 \mathrm{E}-04$ & $6.1415 \mathrm{E}-04$ & $6.6625 \mathrm{E}-04$ & $1.8129 \mathrm{E}-04$ & $1.8359 \mathrm{E}-04$ \\
\hline & & GBP/USD & $1.1997 \mathrm{E}-04$ & $9.5389 \mathrm{E}-04$ & $4.7618 \mathrm{E}-04$ & $1.1637 \mathrm{E}-04$ & $1.1917 \mathrm{E}-04$ \\
\hline & & CSI 300 & $3.0428 \mathrm{E}-03$ & $1.8117 \mathrm{E}-02$ & $1.3661 \mathrm{E}-02$ & $1.6540 \mathrm{E}-03$ & $1.5752 \mathrm{E}-03$ \\
\hline \multirow[t]{5}{*}{1} & \multirow[t]{5}{*}{20} & EUR/USD & $1.8600 \mathrm{E}-06$ & $4.8420 \mathrm{E}-04$ & $6.1201 \mathrm{E}-04$ & $1.7829 \mathrm{E}-06$ & $3.9546 \mathrm{E}-06$ \\
\hline & & GBP/EUR & $1.6370 \mathrm{E}-05$ & $8.1149 \mathrm{E}-04$ & 2.0203E-04 & 1.6140E-05 & $1.7539 \mathrm{E}-05$ \\
\hline & & GBP/JPY & $5.4100 \mathrm{E}-05$ & $5.3093 \mathrm{E}-04$ & $7.7312 \mathrm{E}-04$ & $5.1605 \mathrm{E}-05$ & $5.2294 \mathrm{E}-05$ \\
\hline & & GBP/USD & $3.6530 \mathrm{E}-05$ & 4.1159E-04 & 4.6871E-04 & $3.5787 \mathrm{E}-05$ & $3.5966 \mathrm{E}-05$ \\
\hline & & CSI 300 & $2.3797 \mathrm{E}-03$ & $6.7069 \mathrm{E}-03$ & $8.9285 \mathrm{E}-03$ & $1.4485 \mathrm{E}-03$ & $1.4705 \mathrm{E}-03$ \\
\hline \multirow[t]{5}{*}{1} & \multirow[t]{5}{*}{100} & EUR/USD & $3.7480 \mathrm{E}-05$ & $5.5738 \mathrm{E}-04$ & $8.1022 \mathrm{E}-04$ & $3.5610 \mathrm{E}-05$ & $4.0239 \mathrm{E}-05$ \\
\hline & & GBP/EUR & $1.3290 \mathrm{E}-05$ & $7.2007 \mathrm{E}-04$ & $6.4341 \mathrm{E}-04$ & $1.3271 \mathrm{E}-05$ & $1.5907 \mathrm{E}-05$ \\
\hline & & GBP/JPY & $8.6800 \mathrm{E}-06$ & 7.7477E-04 & $4.3280 \mathrm{E}-04$ & $8.5912 \mathrm{E}-06$ & $1.0554 \mathrm{E}-05$ \\
\hline & & GBP/USD & $2.3800 \mathrm{E}-05$ & $1.8636 \mathrm{E}-04$ & $6.8801 \mathrm{E}-04$ & $2.2758 \mathrm{E}-05$ & $2.4789 \mathrm{E}-05$ \\
\hline & & CSI 300 & $5.1285 \mathrm{E}-03$ & $1.9782 \mathrm{E}-03$ & $2.8437 \mathrm{E}-03$ & $1.6060 \mathrm{E}-03$ & $1.5383 \mathrm{E}-03$ \\
\hline \multirow[t]{5}{*}{100} & \multirow[t]{5}{*}{200} & EUR/USD & $6.6780 \mathrm{E}-05$ & $6.8664 \mathrm{E}-04$ & $5.7217 \mathrm{E}-04$ & $6.5737 \mathrm{E}-05$ & $6.6614 \mathrm{E}-05$ \\
\hline & & GBP/EUR & $4.2900 \mathrm{E}-06$ & $8.3487 \mathrm{E}-04$ & $6.1414 \mathrm{E}-04$ & $4.2544 \mathrm{E}-06$ & $5.0717 \mathrm{E}-06$ \\
\hline & & GBP/JPY & $2.8200 \mathrm{E}-06$ & $6.8156 \mathrm{E}-04$ & $5.2273 \mathrm{E}-04$ & $2.7291 \mathrm{E}-06$ & $5.2139 \mathrm{E}-06$ \\
\hline & & GBP/USD & $2.5410 \mathrm{E}-05$ & $5.8532 \mathrm{E}-04$ & $1.1835 \mathrm{E}-04$ & $2.5375 \mathrm{E}-05$ & $2.4840 \mathrm{E}-05$ \\
\hline & & CSI 300 & $6.5524 \mathrm{E}-03$ & $5.0172 \mathrm{E}-02$ & $4.5828 \mathrm{E}-02$ & $1.1268 \mathrm{E}-03$ & $1.1443 \mathrm{E}-03$ \\
\hline \multirow[t]{5}{*}{260} & \multirow[t]{5}{*}{360} & EUR/USD & $7.2020 \mathrm{E}-05$ & $7.2760 \mathrm{E}-04$ & $4.4519 \mathrm{E}-04$ & $6.9409 \mathrm{E}-05$ & 7.1971E-05 \\
\hline & & GBP/EUR & $1.9310 \mathrm{E}-05$ & $2.0510 \mathrm{E}-04$ & $7.2379 \mathrm{E}-04$ & $1.8586 \mathrm{E}-05$ & $2.0965 \mathrm{E}-05$ \\
\hline & & GBP/JPY & $7.2790 \mathrm{E}-05$ & $1.5105 \mathrm{E}-04$ & $8.6109 \mathrm{E}-04$ & $6.9512 \mathrm{E}-05$ & $7.2097 \mathrm{E}-05$ \\
\hline & & GBP/USD & $7.5100 \mathrm{E}-05$ & $7.2893 \mathrm{E}-04$ & $9.2200 \mathrm{E}-04$ & $7.4866 \mathrm{E}-05$ & $7.3583 \mathrm{E}-05$ \\
\hline & & CSI 300 & 4.6362E-03 & $2.3344 \mathrm{E}-02$ & 3.0649E-02 & $1.7042 \mathrm{E}-03$ & $1.7497 \mathrm{E}-03$ \\
\hline \multirow[t]{5}{*}{400} & \multirow[t]{5}{*}{500} & EUR/USD & $2.7220 \mathrm{E}-05$ & 8.9625E-04 & 4.8093E-04 & $2.6178 \mathrm{E}-05$ & $2.8225 \mathrm{E}-05$ \\
\hline & & GBP/EUR & $5.4620 \mathrm{E}-05$ & $7.3370 \mathrm{E}-04$ & $2.6279 \mathrm{E}-04$ & $5.2996 \mathrm{E}-05$ & $5.5345 \mathrm{E}-05$ \\
\hline & & GBP/JPY & $4.8850 \mathrm{E}-05$ & $1.9753 \mathrm{E}-04$ & $3.6042 \mathrm{E}-04$ & 4.8097E-05 & $4.9049 \mathrm{E}-05$ \\
\hline & & GBP/USD & $3.7620 \mathrm{E}-05$ & $2.5680 \mathrm{E}-04$ & $3.3710 \mathrm{E}-04$ & $3.7423 \mathrm{E}-05$ & $3.8794 \mathrm{E}-05$ \\
\hline & & CSI 300 & $6.5113 \mathrm{E}-03$ & $3.8719 \mathrm{E}-02$ & $4.7354 \mathrm{E}-02$ & $1.1239 \mathrm{E}-03$ & $1.1293 \mathrm{E}-03$ \\
\hline
\end{tabular}


Table 3. The Diebold and Mariano (1995) t-statistics

This table reports the heteroscedasticity- and serial correlation-adjusted $t$-statistics for the Diebold and Mariano (1995) pairwise comparison for out-of-sample forecasts between volatility models. A positive $t$-statistic indicates that the model in the row is preferred to the one in the column and a negative $t$-statistic indicates that the model in the column is preferred. The out-of-sample period is from 8 December 2012 to 15 August 2015 for the exchange rates and from 1 August 2008 to 29 September 2017 for the CSI 300 index. The forecast horizon is from day $t+\tau_{1}$ to day $t+\tau_{2}$.

\begin{tabular}{|c|c|c|c|c|c|c|c|c|c|c|c|c|}
\hline$\tau_{1}$ & $\tau_{2}$ & Model & HSY & EL & HSY & EL & HSY & EL & HSY & EL & HSY & EL \\
\hline \multirow{3}{*}{1} & \multirow{3}{*}{5} & & \multicolumn{2}{|c|}{ EUR/USD } & \multicolumn{2}{|c|}{ GBP/EUR } & \multicolumn{2}{|c|}{ GBP/JPY } & \multicolumn{2}{|c|}{ GBP/USD } & \multicolumn{2}{|c|}{ CSI 300} \\
\hline & & Hybrid & 7.6910 & 1.0911 & 5.9408 & 1.9183 & 3.4773 & 4.6680 & 4.1081 & 1.6847 & 6.3588 & 16.212 \\
\hline & & HSY & & 1.8249 & & 1.3077 & & -1.2881 & & -0.6033 & & 0.6538 \\
\hline \multirow[t]{2}{*}{1} & 20 & Hybrid & 8.7476 & 2.5996 & 4.8397 & 0.2919 & 7.3755 & 0.1594 & 6.703 & 0.3364 & 9.8914 & 7.2365 \\
\hline & & HSY & & 1.8127 & & 0.1300 & & -1.4201 & & 0.1337 & & 0.3963 \\
\hline \multirow[t]{2}{*}{1} & 100 & Hybrid & 5.9177 & 0.9729 & 2.1292 & 1.5571 & 9.9636 & 2.9318 & 2.6785 & 0.3477 & 8.0337 & 10.248 \\
\hline & & HSY & & -0.1497 & & 1.4592 & & -0.8297 & & 1.7061 & & 1.3098 \\
\hline \multirow[t]{2}{*}{100} & 200 & Hybrid & 6.6101 & 9.2975 & 9.5181 & 7.4871 & 9.0246 & 9.4286 & 9.1726 & 6.1150 & 12.097 & 77.671 \\
\hline & & HSY & & -1.0583 & & -0.4127 & & -1.0150 & & -0.4382 & & -1.4875 \\
\hline \multirow[t]{2}{*}{260} & 360 & Hybrid & 2.9623 & 8.9967 & 6.9078 & 7.7334 & 6.5597 & 9.6965 & 2.4652 & 9.0027 & 7.2266 & 87.907 \\
\hline & & HSY & & -0.3716 & & 0.0996 & & 0.7700 & & 0.8243 & & 0.8005 \\
\hline \multirow[t]{2}{*}{400} & 500 & Hybrid & 4.1896 & 6.6620 & 3.0864 & 6.0912 & 2.7686 & 8.6599 & 5.5633 & 9.7639 & 5.7732 & 46.623 \\
\hline & & HSY & & 0.3823 & & 1.0873 & & 1.4280 & & -1.0954 & & 1.1218 \\
\hline
\end{tabular}


Table 4. The superior predicative ability (SPA) test of Hansen (2005)

This table reports the Hansen (2005) SPA test results for out-of-sample volatility forecasts based on the RMSFE. The benchmark model is the Hybrid model. The out-of-sample period is from 8 December 2012 to 15 August 2015 for the exchange rates and from 1 August 2008 to 29 September 2017 for the CSI 300 index. The stationary bootstrapped $p$-values are obtained via 1000 replications.

\begin{tabular}{lcclr} 
Currency & Benchmark Model & Competing Models & $p$-value \\
\hline EUR/USD & Hybrid & HSY & EL & 0.9397 \\
GBP/EUR & Hybrid & HSY & EL & 0.8534 \\
EUR/JPY & Hybrid & HSY & EL & 0.9105 \\
GBP/USD & Hybrid & HSY & EL & 0.8207 \\
CSI 300 & Hybrid & HSY & EL & 0.8545 \\
\hline
\end{tabular}




\section{Table 5. Interval evaluation of volatility forecasts}

This table reports the $p$-values of the Christoffersen (1998) interval forecast evaluation with the null hypothesis of correct coverage $H_{0}: f=5 \%$. These include the likelihood ratio of unconditional coverage $L R_{u c}$, the likelihood ratio of independence $L R_{i n d}$, and the likelihood ratio of conditional coverage $L R_{c c}$. The out-of-sample period is from 8 December 2012 to 15 August 2015 for the exchange rates and from 1 August 2008 to 29 September 2017 for the CSI 300 index.

\begin{tabular}{lllll} 
Currency & Model & $L R_{u c}$ & $L R_{\text {ind }}$ & $L R_{c c}$ \\
\hline EUR/USD & Hybrid & 0.8188 & 0.7859 & 0.6373 \\
& HSY & 0.4762 & 0.4545 & 0.4674 \\
& EL & 0.4670 & 0.3218 & 0.4761 \\
\cline { 2 - 5 } GBP/EUR & Hybrid & 0.6976 & 0.8048 & 0.6720 \\
& HSY & 0.4350 & 0.4098 & 0.3022 \\
& EL & 0.3080 & 0.3243 & 0.3419 \\
\cline { 2 - 5 } GBP/JPY & Hybrid & 0.7610 & 0.8283 & 0.6833 \\
& HSY & 0.4539 & 0.3894 & 0.4163 \\
& EL & 0.3859 & 0.4297 & 0.4156 \\
\cline { 2 - 5 } GBP/USD & Hybrid & 0.7458 & 0.6900 & 0.6210 \\
& HSY & 0.3940 & 0.4534 & 0.3436 \\
& EL & 0.5266 & 0.3011 & 0.4648 \\
\cline { 2 - 5 } CSI 300 & Hybrid & 0.7635 & 0.8685 & 0.6296 \\
& HSY & 0.4400 & 0.4438 & 0.3877 \\
& EL & 0.4209 & 0.3473 & 0.4260 \\
\hline
\end{tabular}


Table 6. Volatility forecasting error analysis

This table reports the average absolute percentage error (APE) in percent of the long- and short-term components of volatility forecasts by the neural network enhanced model (Hybrid), the cyclical model of Harris et al. (2011) model (HSY), and the component GARCH of Engle and Lee (1999)(EL) over different forecasting horizons. The out-of-sample period is from 8 December 2012 to 15 August 2015 for the exchange rates and from 1 August 2008 to 29 September 2017 for the CSI 300 index. The forecast horizon is from day $t+\tau_{1}$ to day $t+\tau_{2}$.

\begin{tabular}{lllllcccc} 
Currency & $\tau_{1}$ & $\tau_{2}$ & HSY LT & HSY ST & EL LT & EL ST & Hybrid LT & Hybrid ST \\
\hline EUR/USD & 1 & 5 & 0.2628 & 34.407 & 0.8229 & 25.877 & 0.0731 & 12.931 \\
& 1 & 20 & 0.9122 & 34.459 & 0.8095 & 24.537 & 0.0483 & 16.326 \\
& 1 & 100 & 4.6594 & 30.516 & 3.2042 & 26.723 & 0.0784 & 12.622 \\
& 100 & 200 & 23.829 & 23.088 & 13.454 & 24.925 & 0.0117 & 8.7000 \\
& 260 & 360 & 20.716 & 29.686 & 19.143 & 26.603 & 0.0260 & 15.738 \\
& 400 & 500 & 28.346 & 23.918 & 21.681 & 23.294 & 0.8634 & 9.7544 \\
\cline { 2 - 8 } GBP/EUR & 1 & 5 & 0.6066 & 26.851 & 0.8923 & 29.145 & 0.0961 & 12.486 \\
& 1 & 20 & 0.4939 & 31.201 & 0.4922 & 26.311 & 0.0806 & 13.170 \\
& 1 & 100 & 3.7014 & 31.629 & 4.4049 & 22.591 & 0.1086 & 11.088 \\
& 100 & 200 & 23.326 & 23.528 & 16.110 & 22.236 & 0.0590 & 10.388 \\
& 260 & 360 & 11.457 & 24.254 & 16.843 & 22.189 & 0.1083 & 11.148 \\
& 400 & 500 & 29.375 & 24.502 & 25.182 & 29.769 & 1.3931 & 8.5424 \\
\cline { 2 - 8 } GBP/JPY & 1 & 5 & 0.4931 & 22.640 & 0.6058 & 27.431 & 0.0310 & 14.133 \\
& 1 & 20 & 0.2197 & 25.788 & 0.8384 & 22.447 & 0.0582 & 15.069 \\
& 1 & 100 & 5.4097 & 26.420 & 3.9443 & 26.874 & 0.1092 & 15.236 \\
& 100 & 200 & 17.045 & 29.865 & 21.506 & 29.998 & 0.0646 & 12.184 \\
& 260 & 360 & 21.970 & 31.153 & 13.065 & 23.162 & 0.0127 & 12.711 \\
& 400 & 500 & 17.467 & 29.353 & 16.698 & 28.497 & 0.8957 & 13.101 \\
\cline { 2 - 8 } GBP/USD & 1 & 5 & 0.4507 & 22.102 & 0.8923 & 26.239 & 0.1089 & 15.924 \\
& 1 & 20 & 1.0454 & 28.054 & 0.4939 & 27.121 & 0.0140 & 14.818 \\
& 1 & 100 & 2.9916 & 26.621 & 4.0927 & 24.109 & 0.0300 & 17.533 \\
& 100 & 200 & 13.030 & 29.968 & 18.911 & 24.768 & 0.0176 & 11.413 \\
& 260 & 360 & 16.254 & 33.174 & 19.835 & 27.936 & 0.0296 & 15.761 \\
& 400 & 500 & 32.017 & 27.588 & 17.707 & 24.676 & 1.4686 & 8.9945 \\
\hline & & & & & & & & \\
CSI 300 & 5 & 0.3607 & 20.813 & 0.5679 & 19.691 & 0.0567 & 10.876 \\
& 1 & 20 & 0.5293 & 23.326 & 0.4962 & 18.570 & 0.0368 & 10.457 \\
& 1 & 100 & 3.2654 & 21.381 & 2.7823 & 18.837 & 0.0609 & 10.317 \\
& 100 & 200 & 14.636 & 20.702 & 12.642 & 18.576 & 0.0300 & 8.2616 \\
& 260 & 360 & 13.291 & 22.744 & 13.666 & 19.076 & 0.0351 & 9.9418 \\
& 400 & 500 & 21.130 & 20.491 & 15.499 & 19.035 & 0.9195 & 7.6124 \\
\hline
\end{tabular}


Table 7. Robustness test: The superior predicative ability (SPA) test of Hansen (2005) on range-based volatility

This table reports the Hansen (2005) SPA test results based on the RMSFE and intraday range-based volatility. The benchmark model is the Hybrid model. The out-of-sample period is from 8 December 2012 to 15 August 2015 for the exchange rates and from 1 August 2008 to 29 September 2017 for the CSI 300 index. The stationary bootstrapped $p$-values are obtained via 1000 replications.

\begin{tabular}{lcclr} 
Currency & Benchmark Model & Competing Models & $p$-value \\
\hline EUR/USD & Hybrid & HSY & EL & 0.9210 \\
GBP/EUR & Hybrid & HSY & EL & 0.8947 \\
GBP/JPY & Hybrid & HSY & EL & 0.9209 \\
GBP/USD & Hybrid & HSY & EL & 0.9145 \\
CSI 300 & Hybrid & HSY & EL & 0.8561 \\
\hline
\end{tabular}


Table 8. Robustness test: The Diebold and Mariano (1995) $t$-statistics with $Q L I K E$ as loss function

This table reports the the heteroscedasticity- and serial correlation-adjusted $t$-statistics for the Diebold and Mariano (1995) pairwise comparison for out-of-sample forecasts when the loss function is QLIKE. A positive $t$-statistic indicates that the model in the row is preferred to that in the column and a negative $t$-statistic indicates that the model in the column is preferred. The out-of-sample period is from 8 December 2012 to 15 August 2015 for the exchange rates and from 1 August 2008 to 29 September 2017 for the CSI 300 index. The forecast horizon is from day $t+\tau_{1}$ to day $t+\tau_{2}$.

\begin{tabular}{|c|c|c|c|c|c|c|c|c|c|c|c|c|}
\hline$\tau_{1}$ & $\tau_{2}$ & Model & HSY & EL & HSY & EL & HSY & EL & HSY & EL & HSY & EL \\
\hline & & & EUR & /USD & GBP & EUR & GBP & /JPY & GBP & USD & CSI & 300 \\
\hline \multirow[t]{2}{*}{1} & \multirow[t]{2}{*}{5} & Hybrid & 9.0459 & 3.5817 & 4.9504 & 7.2039 & 6.9711 & 4.9047 & 8.9148 & 6.1967 & 10.027 & 6.3491 \\
\hline & & HSY & & -0.7051 & & 1.6107 & & 1.3339 & & 1.7412 & & 1.3039 \\
\hline \multirow[t]{2}{*}{1} & \multirow[t]{2}{*}{20} & Hybrid & 4.3829 & 2.8809 & 6.3633 & 2.7023 & 8.1646 & 7.4947 & 5.3155 & 5.0043 & 8.2222 & 5.9992 \\
\hline & & HSY & & 0.3851 & & 1.2689 & & 0.6834 & & 0.8900 & & 1.0731 \\
\hline \multirow[t]{2}{*}{1} & \multirow[t]{2}{*}{100} & Hybrid & 3.2963 & 7.8311 & 5.9995 & 5.9544 & 8.1877 & 5.6722 & 9.4513 & 4.4997 & 8.5917 & 7.8892 \\
\hline & & HSY & & -0.9142 & & 1.0368 & & 1.0711 & & 1.0209 & & 0.8244 \\
\hline \multirow[t]{2}{*}{100} & \multirow[t]{2}{*}{200} & Hybrid & 4.5872 & 6.2260 & 6.8995 & 6.0620 & 5.6261 & 9.7821 & 5.7510 & 8.6084 & 7.1670 & 9.8616 \\
\hline & & HSY & & 0.6481 & & -0.4753 & & 1.1477 & & 0.9843 & & 0.8475 \\
\hline \multirow[t]{2}{*}{260} & \multirow[t]{2}{*}{360} & Hybrid & 9.8869 & 7.5416 & 3.1872 & 7.8132 & 8.9308 & 8.4944 & 4.1960 & 5.9018 & 7.3566 & 8.3682 \\
\hline & & HSY & & 1.8591 & & -0.4481 & & -0.4951 & & 0.0294 & & 0.3530 \\
\hline \multirow[t]{2}{*}{360} & \multirow[t]{2}{*}{500} & Hybrid & 8.5205 & 6.2515 & 7.2465 & 6.0848 & 9.6953 & 7.4726 & 6.8483 & 8.7984 & 9.9444 & 10.133 \\
\hline & & HSY & & 0.1297 & & 0.9564 & & 0.9288 & & 0.8816 & & 0.8778 \\
\hline
\end{tabular}


Table 9. Robustness: The superior predicative ability (SPA) test of Hansen (2005) on range-based volatility with $Q L I K E$ as loss function

This table reports the Hansen (2005) SPA test results for out-of-sample range-based volatility forecasts when the loss function is QLIKE. The benchmark model is the Hybrid model. The out-of-sample period is from 8 December 2012 to 15 August 2015 for the exchange rates and from 1 August 2008 to 29 September 2017 for the CSI 300 index. The stationary bootstrapped $p$-values are obtained via 1000 replications.

\begin{tabular}{lcclr} 
Currency & Benchmark Model & Competing Models & $p$-value \\
\hline EUR/USD & Hybrid & HSY & EL & 0.9406 \\
GBP/EUR & Hybrid & HSY & EL & 0.9781 \\
GBP/JPY & Hybrid & HSY & EL & 0.9382 \\
GBP/USD & Hybrid & HSY & EL & 0.9547 \\
CSI 300 & Hybrid & HSY & EL & 0.8015 \\
\hline
\end{tabular}


Table 10. The Diebold and Mariano t-statistics for volatility forecasts with alternative neural network models

This table reports the heteroscedasticity- and serial correlation-adjusted $t$-statistics for the Diebold and Mariano (1995) pairwise comparison for out-of-sample forecasts when the long-term component is modeled by the ARNN (Hybrid), the LSTM (Hybrid-LSTM), or the GRU (Hybrid-GRU). A positive $t$-statistic indicates that the model in the row is preferred to that in the column and a negative $t$-statistic indicates that the model in the column is preferred. The out-of-sample period is from 8 December 2012 to 15 August 2015 for the exchange rates and from 1 August 2008 to 29 September 2017 for the CSI 300 index. The forecast horizon is from day $t+\tau_{1}$ to day $t+\tau_{2}$.

\begin{tabular}{|c|c|c|c|c|c|c|c|c|c|c|c|c|}
\hline$\tau_{1}$ & $\tau_{2}$ & Model & Hybrid-GRU & Hybrid & Hybrid-GRU & Hybrid & Hybrid-GRU & Hybrid & Hybrid-GRU & Hybrid & Hybrid-GRU & Hybrid \\
\hline \multirow{3}{*}{1} & \multirow{3}{*}{5} & & \multicolumn{2}{|c|}{ EUR/USD } & \multicolumn{2}{|c|}{ GBP/EUR } & \multicolumn{2}{|c|}{ GBP/JPY } & \multicolumn{2}{|c|}{ GBP/USD } & \multicolumn{2}{|c|}{ CSI 300 Index } \\
\hline & & Hybrid-LSTM & 0.6430 & 0.9519 & 0.5518 & 0.5487 & 0.9997 & 0.3690 & 0.6325 & 0.4507 & 1.3837 & 0.8296 \\
\hline & & Hybrid-GRU & & 0.8023 & & 0.4779 & & 0.2660 & & 0.4229 & & 0.8887 \\
\hline \multirow[t]{2}{*}{1} & \multirow[t]{2}{*}{20} & Hybrid-LSTM & 0.5162 & 0.8597 & \multirow[t]{2}{*}{0.6240} & 0.4818 & 0.4985 & 0.6414 & \multirow[t]{2}{*}{0.6554} & 0.7500 & 1.0279 & 0.9104 \\
\hline & & Hybrid-GRU & & 0.8526 & & 0.4066 & & 0.5272 & & 0.7233 & & 0.9308 \\
\hline \multirow[t]{2}{*}{1} & \multirow[t]{2}{*}{100} & Hybrid-LSTM & 0.3111 & 0.5685 & \multirow[t]{2}{*}{0.3524} & 0.2588 & 0.3728 & 1.2665 & \multirow[t]{2}{*}{0.3655} & 0.2386 & 0.5371 & 0.7136 \\
\hline & & Hybrid-GRU & & 0.6190 & & 0.1548 & & 0.8897 & & 0.2241 & & 0.6141 \\
\hline \multirow[t]{2}{*}{100} & \multirow[t]{2}{*}{200} & Hybrid-LSTM & 0.2682 & 0.6827 & \multirow[t]{2}{*}{0.1135} & 1.0738 & 0.0454 & 0.9087 & \multirow[t]{2}{*}{0.4376} & 1.2863 & 0.5370 & 0.8221 \\
\hline & & Hybrid-GRU & & 0.7199 & & 0.9510 & & 0.8056 & & 0.7759 & & 0.6884 \\
\hline \multirow[t]{2}{*}{260} & \multirow[t]{2}{*}{360} & Hybrid-LSTM & 0.0835 & 0.3507 & \multirow[t]{2}{*}{0.1526} & 0.8456 & 0.2570 & 0.6446 & \multirow[t]{2}{*}{0.1704} & 0.2582 & 0.1347 & 0.3264 \\
\hline & & Hybrid-GRU & & 0.2540 & & 0.5557 & & 0.5404 & & 0.1768 & & 0.3197 \\
\hline \multirow[t]{2}{*}{400} & \multirow[t]{2}{*}{500} & Hybrid-LSTM & 0.2133 & 0.4310 & \multirow[t]{2}{*}{0.2690} & 0.3390 & 0.1983 & 0.3314 & \multirow[t]{2}{*}{0.1694} & 0.7197 & \multirow[t]{2}{*}{0.4492} & 0.5110 \\
\hline & & Hybrid-GRU & & 0.3541 & & 0.2397 & & 0.3066 & & 0.4847 & & 0.4884 \\
\hline
\end{tabular}


Table 11. Economic value of volatility forecasts

This table reports the annualized excess return, the Sharpe ratio (SR), and the certainty equivalent return (CER) of portfolios constructed from one of the exchange rates or the stock index. Volatility forecasts are generated from different component models, and $\gamma$ represents investor risk aversion level.

\begin{tabular}{|c|c|c|c|c|c|c|c|c|c|c|}
\hline & & \multicolumn{3}{|c|}{$\gamma=3$} & \multicolumn{3}{|c|}{$\gamma=6$} & \multicolumn{3}{|c|}{$\gamma=9$} \\
\hline & & Return & SR & CER & Return & SR & CER & Return & SR & CER \\
\hline \multirow[t]{3}{*}{ EUR/USD } & Hybrid & 6.6372 & 0.3777 & 3.2899 & 5.7058 & 0.3476 & 1.8707 & 4.7349 & 0.1740 & 1.3264 \\
\hline & HSY & 5.9258 & 0.3372 & 2.8888 & 5.4874 & 0.3197 & 1.5864 & 4.1576 & 0.1482 & 0.7013 \\
\hline & $\mathrm{EL}$ & 5.8304 & 0.3289 & 2.3762 & 4.7976 & 0.3025 & 1.3911 & 4.0602 & 0.1034 & 0.4166 \\
\hline \multirow[t]{3}{*}{ GBP/EUR } & Hybrid & 6.9312 & 0.3892 & 3.2039 & 5.7600 & 0.2950 & 2.3731 & 4.7689 & 0.1892 & 0.7766 \\
\hline & HSY & 6.6279 & 0.3186 & 2.6733 & 5.0146 & 0.2826 & 2.0259 & 3.7181 & 0.1621 & 0.6743 \\
\hline & EL & 6.5132 & 0.3081 & 2.3023 & 4.9048 & 0.2816 & 1.5574 & 3.6557 & 0.1294 & 0.3607 \\
\hline \multirow[t]{3}{*}{ GBP/JPY } & Hybrid & 6.8439 & 0.3727 & 3.3161 & 5.1965 & 0.3727 & 2.3464 & 4.7266 & 0.1594 & 0.9928 \\
\hline & HSY & 6.5757 & 0.3370 & 2.5486 & 4.7453 & 0.3475 & 1.5245 & 4.5637 & 0.1390 & 0.7064 \\
\hline & EL & 5.9690 & 0.3063 & 2.3624 & 4.6618 & 0.3017 & 1.4917 & 4.4927 & 0.1018 & 0.5662 \\
\hline \multirow[t]{3}{*}{ GBP/USD } & Hybrid & 6.8289 & 0.3899 & 2.8545 & 5.5223 & 0.3645 & 2.2893 & 4.7829 & 0.1842 & 1.2356 \\
\hline & HSY & 6.5716 & 0.3360 & 2.5614 & 5.3603 & 0.3079 & 1.9119 & 4.3187 & 0.1771 & 0.2664 \\
\hline & $\mathrm{EL}$ & 6.3060 & 0.3294 & 2.4725 & 5.3566 & 0.3050 & 1.2160 & 3.9778 & 0.1739 & 0.2365 \\
\hline \multirow[t]{3}{*}{ CSI 300} & Hybrid & 5.4872 & 0.3078 & 2.5399 & 4.9036 & 0.2990 & 1.9427 & 4.2188 & 0.1471 & 0.9372 \\
\hline & HSY & 4.7624 & 0.2494 & 1.8783 & 3.6096 & 0.2355 & 1.2770 & 3.2926 & 0.1224 & 0.4168 \\
\hline & EL & 3.8337 & 0.2119 & 1.6364 & 3.0017 & 0.1951 & 0.9287 & 2.5328 & 0.0791 & 0.2556 \\
\hline
\end{tabular}


Figure 1. Volatility decomposition

In (a), (c), and (e) the blue curve represents the daily realized volatility for EUR/USD from 27 September 2009 to 7 December 2012, and the red curve is the long-run component obtained via the wavelet transform with decomposition level 7, 4, and 1, respectively. In (b), (d), and (f) the blue curve is the short-run component of the realized volatility obtained as the difference between the realized volatility and the corresponding long-run component in (a), (c), and (e), respectively.

(a)

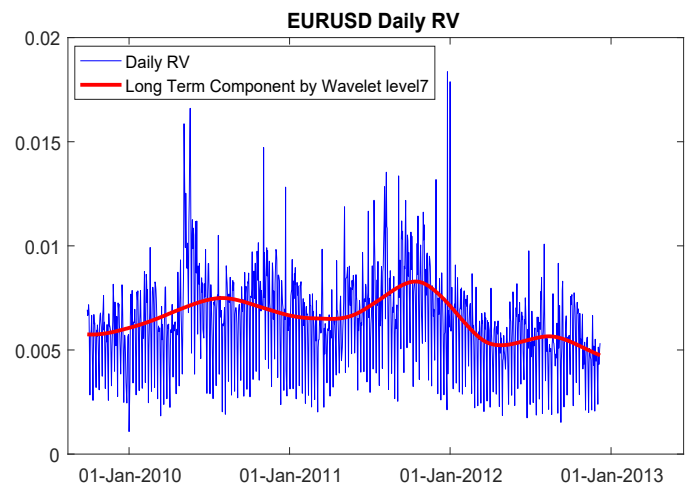

(c)

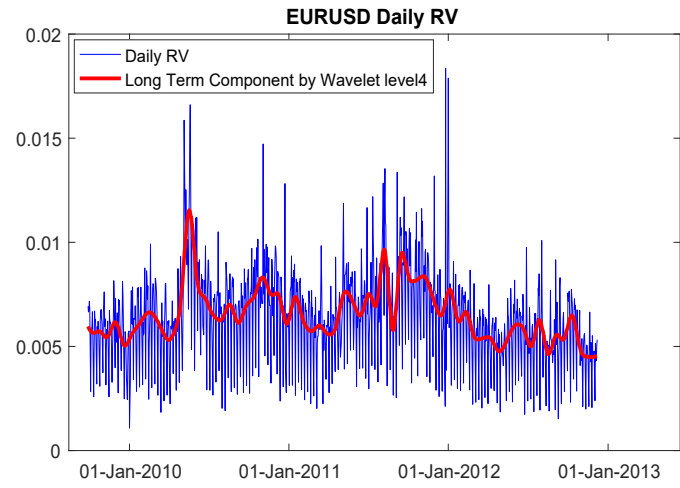

(e)

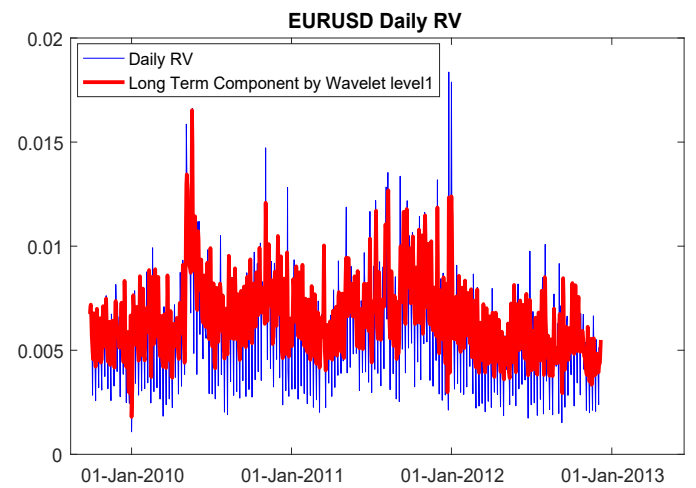

(b)

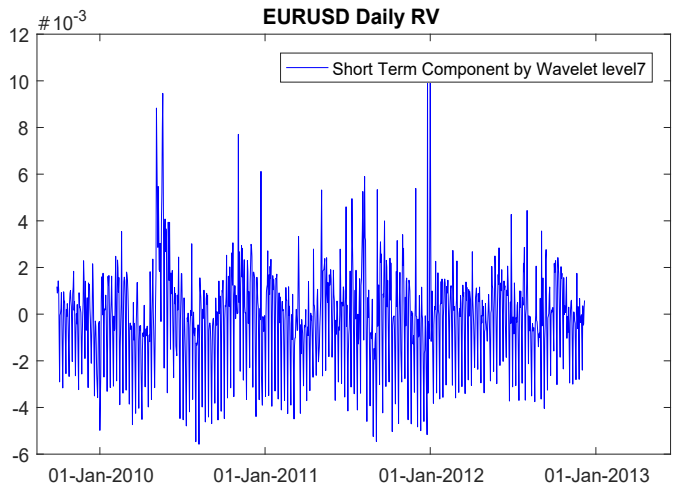

(d)

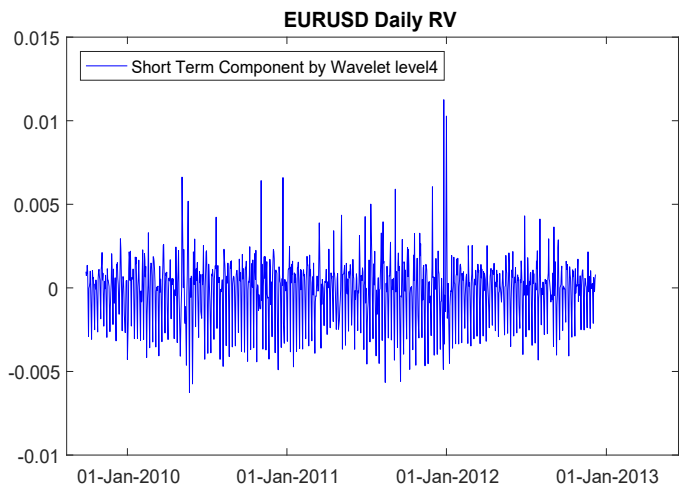

(f)

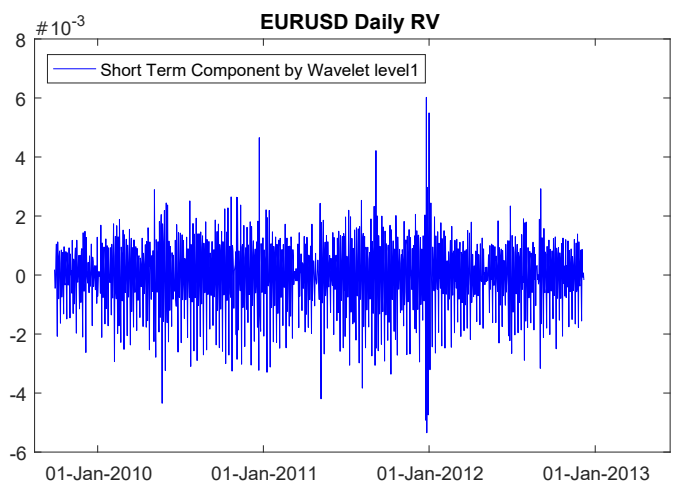


Figure 2. Autocorrelation of daily realized volatility

This figure plots the autocorrelation of the exchange rates and CSI 300 index for up to 30 lags.
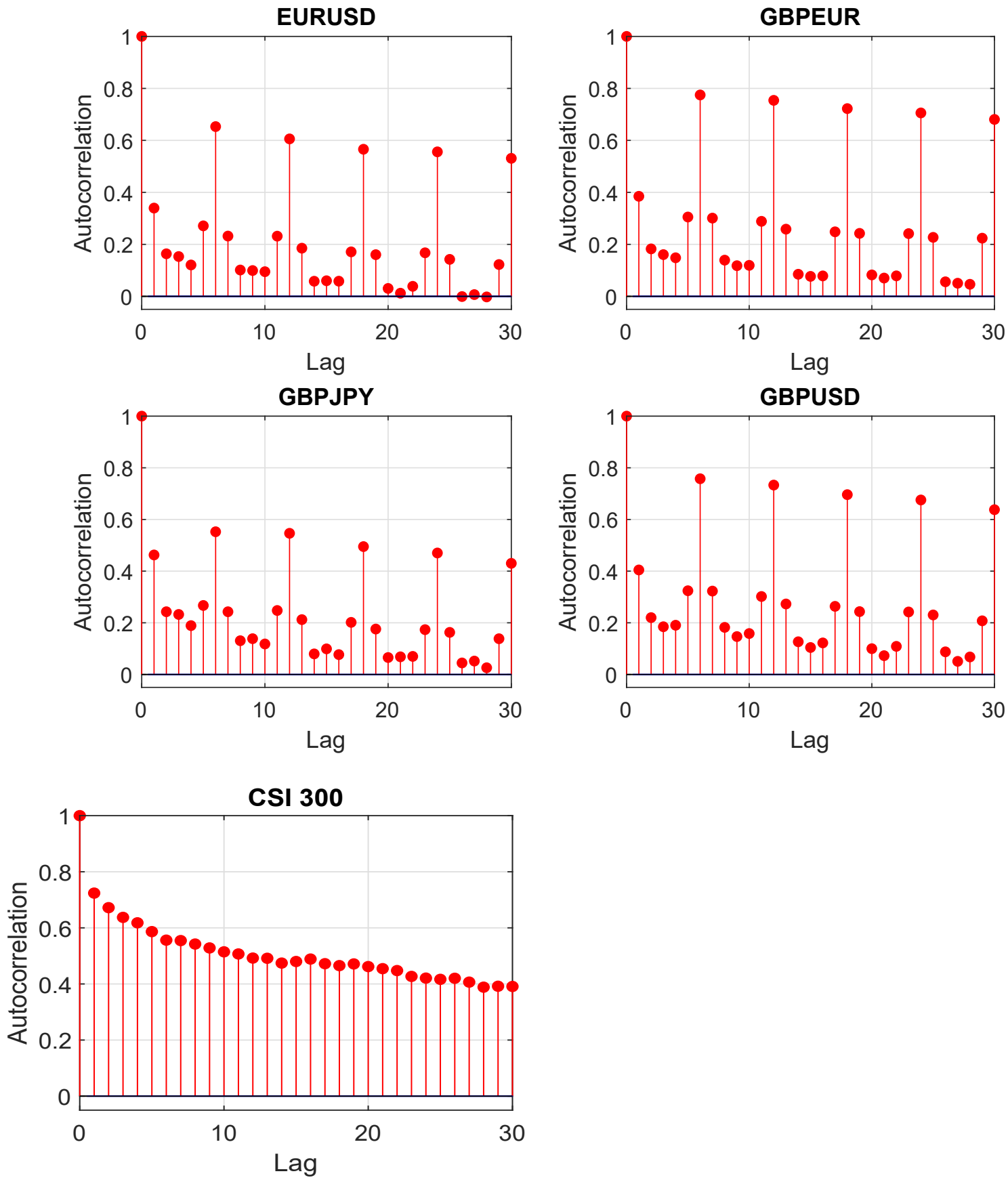
Figure 3. Volatility forecasting error break-down

In (a) and (b) we plot the average absolute percentage error (APE) of the short- and long-term components, respectively, of the forecasted volatility from the Harris et al. (2011) model (HSY), the component GARCH model of Engle and Lee (1999)(EL), and the Hybrid model for EUR/USD. The out-of-sample period is from 8 December 2012 to 15 August 2015.

(a) Short-term component

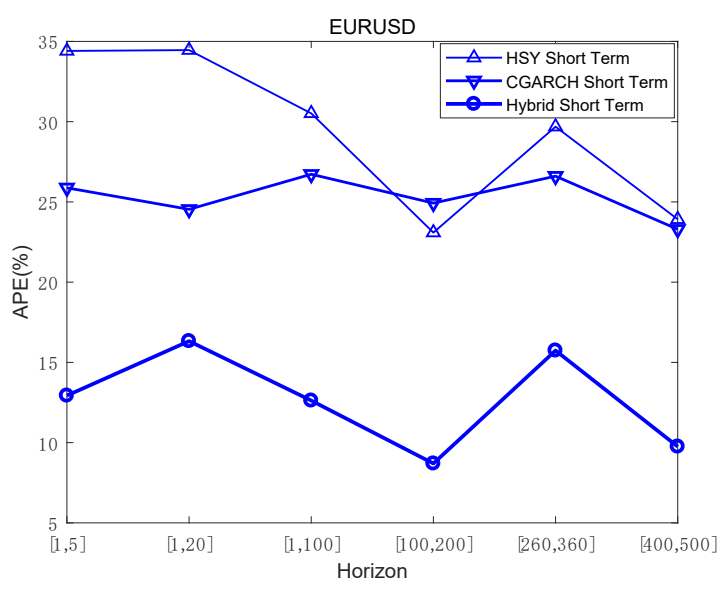

(b) Long-term component

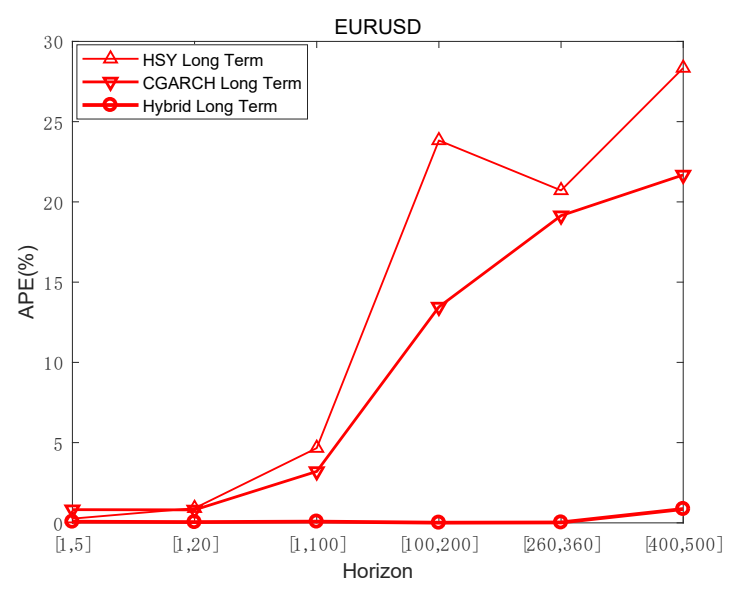

